\title{
Multiple attractors of host-parasitoid models with integrated pest management strategies: Eradication, persistence and outbreak
}

\author{
Sanyi Tang a,*, Yanni Xiao ${ }^{\mathrm{b}, 1}$, Robert A. Cheke ${ }^{\mathrm{c}}$

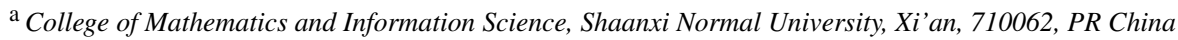 \\ ${ }^{\mathrm{b}}$ Department of Applied Mathematics, Xi'an Jiaotong University, Xi'an, 710049, PR China \\ ${ }^{\mathrm{c}}$ Natural Resources Institute, University of Greenwich at Medway, Central Avenue, Chatham Maritime, Chatham, Kent, ME4 4TB, UK
}

Received 9 September 2006

Available online 8 December 2007

\begin{abstract}
Host-parasitoid models including integrated pest management (IPM) interventions with impulsive effects at both fixed and unfixed times were analyzed with regard to host-eradication, host-parasitoid persistence and host-outbreak solutions. The host-eradication periodic solution with fixed moments is globally stable if the host's intrinsic growth rate is less than the summation of the mean host-killing rate and the mean parasitization rate during the impulsive period. Solutions for all three categories can coexist, with switch-like transitions among their attractors showing that varying dosages and frequencies of insecticide applications and the numbers of parasitoids released are crucial. Periodic solutions also exist for models with unfixed moments for which the maximum amplitude of the host is less than the economic threshold. The dosages and frequencies of IPM interventions for these solutions are much reduced in comparison with the pest-eradication periodic solution. Our results, which are robust to inclusion of stochastic effects and with a wide range of parameter values, confirm that IPM is more effective than any single control tactic.

(c) 2007 Elsevier Inc. All rights reserved.
\end{abstract}

Keywords: Nicholson-Bailey model; IPM; Economic threshold; Impulsive difference equations; Coexistence; Host outbreak

\section{Introduction}

Biological control is a component of an integrated pest management (IPM) strategy (Greathead, 1992; Parker, 1971). It is defined as the reduction of pest populations by natural enemies and typically involves an active human role, such as augmentation which involves the supplemental release of natural enemies. Relatively few natural enemies may be released at a critical time of the season (inoculative release) or millions may be released (inundative release) when insufficient reproduction of released natural enemies is likely to occur and pest control will be achieved exclusively by the released individuals themselves. Examples of inoculative release occur in greenhouse production of several crops. Periodic releases of

\footnotetext{
* Corresponding author.

E-mail addresses: sanyitang219@hotmail.com, sytang@snnu.edu.cn (S. Tang).

${ }^{1}$ Research supported by National Natural Science Foundation of China (NSFC 10701062, and 10726040).
}

the parasitoid Encarsia formosa are used to control greenhouse whitefly, and the predatory mite Phytoseiulus persimilis is used for controlling the two-spotted spider mite Tetranychus urticae (Hoffmann and Frodsham, 1993). An example of inundative release was the use of the wasp Epidinocarsis lopezi to control the Cassava Mealy Bug Phenacocus manihoti in more than 16 African countries (Neuenschwander and Herren, 1988).

If all other integrated pest management tactics including biological, cultural, physical and mechanical control are unable to keep an insect pest population below an economic threshold (Fig. 1), then the use of an insecticide to control the pest and prevent economic loss is justified. In most cropping systems, insecticides are still the principal means of controlling pests once the economic threshold has been reached. They can be relatively cheap and are easy to apply, fast-acting, and in most instances can be relied on to control the pests (Hoffmann and Frodsham, 1993).

However, overuse of a single control tactic is discouraged to avoid or delay the development of resistance by the pest to the control tactic, to minimize damage to non-target organisms, 


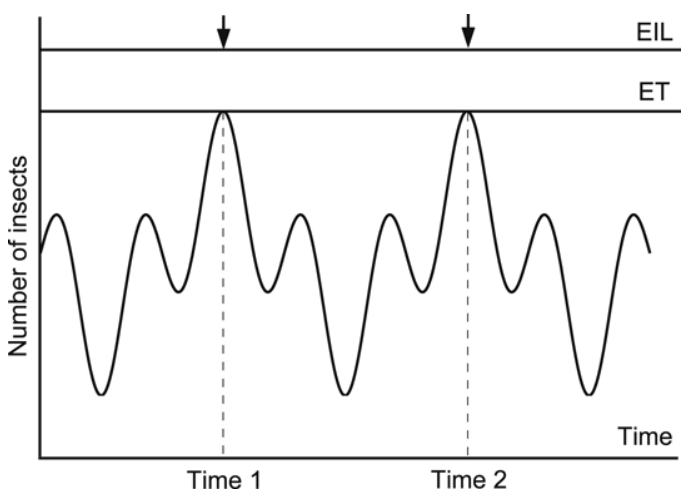

Fig. 1. Economic Injury Level (EIL) = lowest population density that will cause economic damage. Economic Threshold $(E T)=$ population density at which the control measures should be introduced to prevent an increasing pest population from reaching the economic injury level. The arrows indicate points when pest levels exceeded the economic threshold and an IPM strategy would be applied.

and to preserve the quality of the environment. IPM has been shown by experiments (Van Lenteren, 1995, 2000; Van Lenteren and Woets, 1988; Flint, 1987; Pedigo, 1996; Pedigo and Higley, 1992) to be more effective than classical methods (such as biological or chemical control only). An important concept in IPM is that of the economic threshold (ET) (Fig. 1). An economic threshold is usually defined as the number of insect pests in the field when control actions must be taken to prevent the economic injury level (EIL) from being reached and exceeded, where the EIL is the lowest pest population density that will cause economic damage. For an IPM strategy, action must be taken once a critical density of pests is observed in the field so that the EIL is not exceeded. That is, sufficient lead time is needed between the time when an economic density is observed and the time when a treatment is actually applied in the field. Therefore ET must be less than the EIL.

In practice, determining the EIL and ET is generally a complex matter based on detailed investigations of pest ecology as it relates to bioclimatology, predation, diseases, the effect of host-plant resistance and the environmental consequences of applied control interventions (Metcalf and Luckman, 1975). One of the most important questions in IPM is how many natural enemies should be released and what fraction of the insect pest population should be killed to avoid economic damage when the pest population reaches or exceeds the ET level. In many cases, the most effective release rate or spraying rate has not been identified as it will vary depending on crop type and target host density.

Undoubtedly, mathematical modelling is one of the key tools for estimating and predicting population densities (Barclay, 1982; Barlow et al., 1996). Recently, continuous predator-prey models concerning IPM strategies have been developed and investigated (Tang and Chen, 2004; Tang and Cheke, 2005; Tang et al., 2005). Several biologically desirable solutions including a pest-eradication periodic solution and positive periodic solutions with maximum pest amplitude below ET were obtained analytically. These results can help us to design a control strategy such as deciding on the dosage and frequency of insecticide applications and the preferred timings for releasing natural enemies.

However, a common feature among many insect species is that they exhibit discrete population generations. With this scenario the use of continuous-time models to describe the population dynamics of a species becomes questionable, so when populations have discrete and synchronized generations, discrete host-parasitoid models are much more realistic. These models are usually described by a pair of coupled firstorder difference equations (Hassell and May, 1973; Hassell, 1978, 1984, 2000a,b). It has been shown that for the classic Nicholson-Bailey model (Nicholson and Bailey, 1935) for a two species host-parasitoid system with discrete generations, the slightest perturbation away from its steady state results in diverging oscillations of the population sizes of both host and parasitoid. Although unstable, this model inspired many investigations into more complex host-parasitoid systems (Hassell and May, 1973; Broadhead and Cheke, 1975; Beddington et al., 1975; Hassell, 1978, 2000b).

The main purposes of the present paper are to extend the classic Nicholson-Bailey model by addition of a fraction for the survival rate, inside or outside their hosts, of parasitoids from one generation to the next, and to investigate the effect of an IPM strategy on this expanded model. The parasitoid intergenerational survival rate can be affected by several different factors such as immigration from outside the local area, parasitoid overwintering survival and parasitism of an alternate insect pest which is not modelled explicitly. On the one hand, most parasitoids are highly mobile winged insects, such as hymenopterous wasps and dipteran flies, and most of their hosts are insect stages that are partially or completely immobile (e.g. caterpillars, eggs), so when the parasitization takes place the hosts are largely sedentary. One the other hand, some parasitoids such as Sphecophaga vesparum (Curtis) can overwinter for several generations. $S$. vesparum is a parasitoid of some social wasps (Vespinae) and was the first biological control agent introduced against wasps (Donovan and Read, 1987). It has been released throughout much of New Zealand since early 1985 (Donovan and Read, 1987; Beggs et al., 1996; Donovan, 1991). Parasitoid larvae pupate forming one of three types of cocoon (Donovan, 1991), one type being tough, yellow (overwintering) cocoons that produce winged females or males and which may spend up to 4 years in a dormant state before emerging. Another example is Echthromorpha intricatoria which is capable of overwintering as pupae within the pupae of the New Zealand red admiral butterfly Bassaris gonerilla and thus can attack all generations of B. gonerilla (Barron et al., 2004).

Here we investigate what affects the incorporation of parasitoid intergenerational survival rates, the periodicity of insecticide spraying and the augmentation of natural enemies have on the dynamics of the classical Nicholson-Bailey host-parasite system. Firstly, we suggest an impulsive difference system to model the process of periodic releases of natural enemies and spraying pesticides or trapping the pest to eradicate it. We analyze the global stability of the so-called pest-eradication periodic solution, and show that it 
is globally stable if the host's intrinsic growth rate is less than the summation of the mean host-killing rate and mean parasitization rate during the impulsive period. Numerical investigations imply that the dynamical behaviour of a system with fixed moments is dominated by several typical periodic solutions such as host-eradication, host-parasitoid persistence and host-outbreak periodic solutions, which can coexist. This indicates that the final state of the host population depends on its initial conditions, which has been confirmed from the basins of attraction of these stable attractors. The switchlike behaviour is another important characteristic of a system with fixed moments, i.e. small random perturbations can cause state transitions from a host-outbreak solution to a host-parasitoid periodic solution, which shows that varying dosages of insecticide applications and differing numbers of natural enemies released play key roles in pest control. Analysis of this simple model suggests that the role of multiple attractors in the response to IPM strategies should be examined in more realistic host-parasitoid models.

Secondly, we describe an impulsive difference system which models impulsive effects, such as releasing natural enemies and spraying pesticides against the pest or trapping the pest, introduced when the pest population reaches the ET (Fig. 1), aimed at keeping the density of the host population below the ET and investigate its stability properties. Our results show that there exist stable periodic solutions such that the maximum amplitude of the host population does not exceed the given ET. Comparing this type of periodic solution with hosteradication periodic solutions, we conclude that the required dosages and frequencies of insecticide applications are reduced if the density of the host population is only kept below ET rather than being eradicated. However, bifurcation analysis implies that the dynamical behaviour of the system with unfixed moments is much more complex than that of the system with fixed moments. The former is dominated by period doubling bifurcations and chaotic solutions while the latter is dominated by periodic solutions. Theoretically, our results confirm that IPM is more effective than any single control tactic. In practice, the modelling methods and results presented here represent an important step in the design of appropriate IPM strategies.

\section{Host-parasitoid models including parasitoid intergener- ational survival rates}

Traditionally, there have been two distinct starting points for exploring the dynamics of host-parasitoid or predator-prey models, each with its own adherents. Lotka-Volterra models (Lotka, 1920; Volterra, 1931) start with the assumption that generations of the interacting populations overlap completely and that birth and death processes are continuous. Recently, the continuous Lotka-Volterra model with or without impulsive effects concerning IPM strategies has been extensively studied (Grasman et al., 2001; Tang and Chen, 2004; Tang and Cheke, 2005; Tang et al., 2005). Synchronized and discrete generations of both host and parasitoid make it easy to formulate the dynamics of such interacting predator-prey populations in terms of simple mathematical models (Hassell,
1978). Host-parasitoid models are usually described by a pair of coupled first-order difference equations (Hassell, 2000b; Hassell and May, 1973, 1974; May, 1978, 1985; May et al., 1981; May and Hassell, 1988).

A classic discrete-generation host-parasitoid interaction was developed by Nicholson and Bailey (1935):

$$
\left\{\begin{array}{l}
H_{n+1}=H_{n} \exp \left[r-a P_{n}\right], \\
P_{n+1}=H_{n}\left[1-\exp \left(-a P_{n}\right)\right],
\end{array}\right.
$$

where $H_{n}$ and $P_{n}$ are the density of hosts and parasitoids in generation $n . r$ is the intrinsic growth rate of the host population in the absence of parasitoids. The parameter $a$ is a measure of the parasite's searching efficiency, and the term $\exp \left(-a P_{n}\right)$ is the probability that a host individual escapes parasitism. Thus, a particular host individual is parasitized and converted with probability $\left[1-\exp \left(-a P_{n}\right)\right]$ into parasite individuals. It is well known that the Nicholson-Bailey model has a positive fixed point and it is never stable, which means that the slightest perturbation leads to divergent oscillations, but this instability contrasts with the fact that many hosts and parasitoids coexist in nature. A number of stabilizing factors such as spatial heterogeneity, non-random search, density-dependent growth of the host, functional responses of the parasitoid, and mutual interference among searching parasitoids were put forward in order to stabilize coexistence in single host-single parasitoid systems (Hassell and May, 1973; Beddington et al., 1975, 1978; Hassell, 1978; May et al., 1981; Hassell, 2000b). Taking into account an intergenerational survival rate for the parasitoid, we have the following extended Nicholson-Bailey model:

$$
\left\{\begin{array}{l}
H_{n+1}=H_{n} \exp \left[r-a P_{n}\right], \\
P_{n+1}=H_{n}\left[1-\exp \left(-a P_{n}\right)\right]+\delta P_{n},
\end{array}\right.
$$

where $\delta \geq 0$ denotes the density-independent survival of the parasitoid at generation $n$. This could represent immigration from outside the local area, releases of biological control agents, and increased population growth rate of the parasitoid caused by parasitism of an alternate insect pest which is not modelled explicitly (Briggs and Borer, 2005; Dwyer et al., 2000; Stone and Hart, 1999; White and Wilson, 1999).

Linearized local stability analysis shows that the fixed point

$$
\left(H_{*}, P_{*}\right)=\left(\frac{(1-\delta) r}{a(1-\exp (-r))}, \frac{r}{a}\right)
$$

of model (2.2) is also unstable. However, the main purpose of the present paper is to investigate the effect of periodic releases of the parasitoid (such as periodic releases of Encarsia formosa used to control greenhouse whitefly, and the predatory mite Phytoseiulus persimilis used for controlling the twospotted spider mite Tetranychus urticae) and periodic spraying of insecticides on model (2.2), and design control strategies for when the density of the host population reaches the ET. In what follows, the model (2.2) has been extended through two different impulsive effects, fixed or unfixed moment.

\section{Host-parasitoid model with periodic impulsive effects}

In this section, we will extend model (2.2) by introducing a periodic IPM strategy such as periodic releases of natural 
enemies (Parker, 1971) or spraying pesticide at a critical time and examine the consequences of population densities changing very rapidly. For instance, impulsive reduction of the pest population is possible by trapping the pests and/or by poisoning them with chemicals. An impulsive increase of the parasitoid population density can be achieved by laboratorybased breeding followed by releases into the field. So, we investigate what happens when combinations of biological (natural enemies), cultural (trapping), and chemical (killing) tactics that eradicate the pest to extinction, are used and, in addition, determine which is the most efficient strategy within an IPM context. To this end, let us assume that at every $q$ th generation the system (2.2) is subject to a perturbation which incorporates a proportional decrease of the insect pest and proportional increase of the parasitoid and an introduction constant $\tau$ for the parasitoid which does not depend on the sizes of the populations $P_{q k}$. After the perturbation at step $q k$ the size of the population $H_{q k}$ and $P_{q k}$ become

$H_{q k^{+}}=\left(1-q_{1}\right) H_{q k}, \quad P_{q k^{+}}=\left(1+q_{2}\right) P_{q k}+\tau$

where $q$ is a positive integer and $k=1,2, \ldots, 1-q_{1}$ denotes the proportionate survival rate after spraying insecticide, and $q_{2}$ is a proportion representing the release rate of the parasitoid at generation $q k$, and $\tau$ is a releasing constant which does not depend on the density of parasitoids. $H_{q k}$ and $P_{q k}$ are the densities of the host and parasitoid at generation $q k$ before the impulsive perturbations, and $\mathrm{H}_{q k^{+}}$and $P_{q k^{+}}$are the densities of the host and parasitoid at generation $q k$ after the impulsive perturbations.

The control strategies include both the proportional and constant releasing of natural enemies and the killing of the insect pest with insecticide or by trapping. In combination with system (2.2), we have the following system:

$$
\left\{\begin{array}{l}
H_{n+1}=H_{n} \exp \left[r-a P_{n}\right], \\
P_{n+1}=H_{n}\left[1-\exp \left(-a P_{n}\right)\right]+\delta P_{n}, \\
H_{q k^{+}}=\left(1-q_{1}\right) H_{q k}, \\
P_{q k^{+}}=\left(1+q_{2}\right) P_{q k}+\tau,
\end{array}\right\} \quad k=1,2, \ldots, 1,2, \ldots,
$$

where $q$ is a fixed positive integer and denotes the period of the impulsive effect, which means that an integrated control strategy should be applied when $n$ is an integer multiple of $q$. The initial conditions are $\left(H_{0^{+}}, P_{0^{+}}\right)=\left(H_{0}, P_{0}\right)$. Here for convenience we denote the initial density after an impulsive perturbation.

\subsection{Existence and global stability of host-eradication periodic solutions}

In order to consider the existence and stability of hosteradication periodic solutions of system (3.1), we first consider the following simple system:

$\left\{\begin{array}{l}P_{n+1}=\delta P_{n}, \quad n=0,1,2, \ldots, \\ P_{q k^{+}}=\left(1+q_{2}\right) P_{q k}+\tau, \quad k=1,2, \ldots, \\ P_{0}^{+}=P_{0} .\end{array}\right.$

It follows from the periodicity of system (3.2) that the solution $P_{n}$ can be defined at impulsive subinterval $n \in\left[q s^{+}, q(s+1)\right)$ with $s=0,1,2, \ldots$, and $n=q s^{+}$means that we take the density of parasitoids after an impulsive perturbation as the initial value in this interval.

For any $n \in\left[q s^{+}, q(s+1)\right)$, it follows from system (3.2) that

$P_{n+1}=\delta^{n+1-q s} P_{q s^{+}}$.

If $n=q(s+1)-1$ then we have

$P_{q(s+1)}=\delta^{q} P_{q s^{+}}$

and it follows from impulsive conditions that we have

$P_{q(s+1)^{+}}=\left(1+q_{2}\right) P_{q(s+1)}+\tau=\left(1+q_{2}\right) \delta^{q} P_{q s^{+}}+\tau$.

Therefore, the existence of a periodic solution of system (3.2) with period $q$ implies that the difference equation (3.4) has a steady state, i.e.

$P_{0}=\left(1+q_{2}\right) \delta^{q} P_{0}+\tau$

which implies that $P_{0}=\frac{\tau}{1-\left(1+q_{2}\right) \delta^{q}}$, and the following condition is sufficient to guarantee the existence and stability of the periodic solution of system (3.2)

$f_{1} \doteq 1-\left(1+q_{2}\right) \delta^{q}>0$.

Any solution of system (3.2) has the following property:

Lemma 3.1. If inequality (3.5) holds true, then system (3.2) has a positive periodic solution $P_{n}^{*}$ and for every solution $P_{n}$ of (3.2) we have $\left|P_{n}-P_{n}^{*}\right| \rightarrow 0$ as $n \rightarrow \infty$. Where $P_{n}^{*}=\delta^{n-q s} P_{0}, n \in\left[q s^{+}, q(s+1)\right), s=0,1,2, \ldots$, and $P_{0}=\frac{\tau}{1-\left(1+q_{2}\right) \delta^{q}}$.

Therefore, we obtain the complete expression for the 'hosteradication' periodic solution of system (3.1) over the interval $n \in\left[q s^{+}, q(s+1)\right), s=0,1,2, \ldots$, and

$\left(0, P_{n}^{*}\right)=\left(0, \frac{\tau \delta^{n-q s}}{1-\left(1+q_{2}\right) \delta^{q}}\right)$,

and we have the following main theorem of this section for system (3.1).

Theorem 3.1. Let $\left(H_{n}, P_{n}\right)$ be any solution of (3.1). Then $\left(0, P_{n}^{*}\right)$ is globally asymptotically stable in the first quadrant provided that

$r<\frac{1}{q} \ln \left(\frac{1}{1-q_{1}}\right)+\frac{1}{q} \frac{a \tau\left(1-\delta^{q}\right)}{\left(1-\left(1+q_{2}\right) \delta^{q}\right)(1-\delta)}$.

The proof of Theorem 3.1 is given in the Appendix.

In particular, let $q_{2}=0$ and $\tau=0$ (chemical control only), and the host-eradication periodic solution (3.6) is globally stable $((0,0)$ in this case $)$ if the intrinsic growth rate of the host population satisfies

$r<\frac{1}{q} \ln \left(\frac{1}{1-q_{1}}\right)$

which means that if the intrinsic growth rate is less than the mean host-killing rate due to an insecticide application over period $q$, then the host population eventually goes to extinction. 


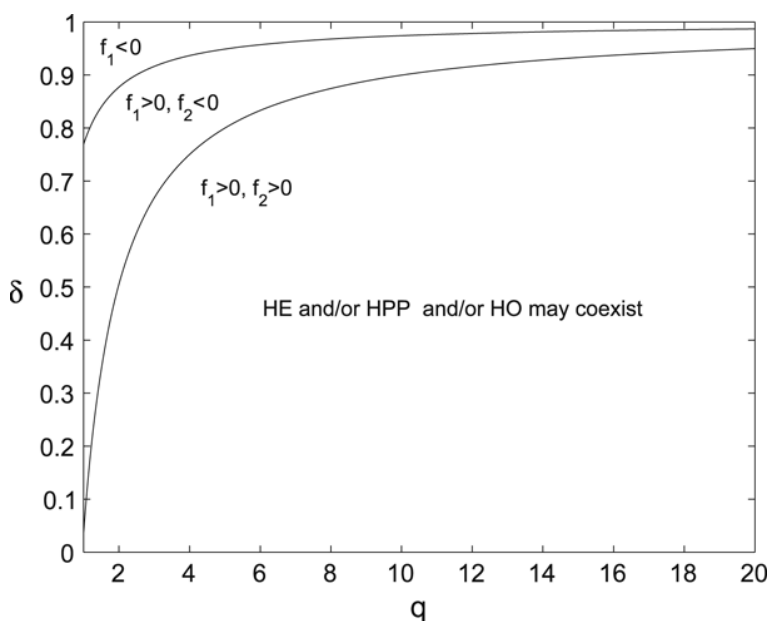

Fig. 2. The $q-\delta$ parameter plane demarcated with the stability of the hosteradication periodic solution. $\left(0, P_{n}^{*}\right)$ is globally stable if $f_{1}>0$ and $f_{2}<0$. The dynamic behaviour becomes very complex if $f_{2}>0$. In this region stable solutions including the HE periodic solution, HPP, and HO solutions and two or all of them coexist. The other parameters are fixed as $r=1.8, a=0.15, q_{1}=$ $0.2, \tau=10$ and $q_{2}=0.3$.

Similarly, let $q_{1}=0$ (biological control only), then the condition which guarantees the global stability of the hosteradication periodic solution becomes:

$r<\frac{1}{q} \frac{a \tau\left(1-\delta^{q}\right)}{\left(1-\left(1+q_{2}\right) \delta^{q}\right)(1-\delta)}$

which means that if the intrinsic growth rate is less than the mean parasitization rate over period $q$, then the host population will become extinct eventually.

However, for an IPM strategy $\left(q_{1}>0, q_{2} \geq 0\right.$ and $\tau>0$ here) inequality (3.7) indicates that if the intrinsic growth rate of the host population is less than the summation of the mean killing rate and the mean parasitization rate over period $q$, then the host population will tend to zero. Theoretically, this confirms that an IPM strategy is more effective than any single control strategy.

If we denote

$f_{2} \doteq q r-\ln \left(\frac{1}{1-q_{1}}\right)-\frac{a \tau\left(1-\delta^{q}\right)}{\left(1-\left(1+q_{2}\right) \delta^{q}\right)(1-\delta)}$,

then the global stability region of the host-eradication periodic solution is determined by $f_{1}>0$ and $f_{2}<0$. One example shown in Fig. 2 shows the existence and stability region of the host-eradication periodic solution in the parameter $q-\delta$ plane. Stability of the host-eradication periodic solution is promoted by high values of parasitoid intergenerational survival rate, $\delta$, and by low values of the impulsive frequency $q$.

Extensive numerical analysis including bifurcation diagrams show that when $f_{2}>0$ there are at least three typical types of solutions according to the evolution of the host population: (a) host-eradication (HE) periodic solution when the parasitoid population periodically oscillates and the host population dies out; (b) host-parasitoid persistence (HPP) solutions, when both species oscillate with positive densities in all generations and the host population oscillates with relatively small amplitudes (for example the maximum amplitude does not exceed ET). For more details of the definition of persistence see Hastings and Higgins (1994); and (c) host-outbreak (HO) or peak-to-peak solutions when the host population has periodic or irregular outbreaks with relatively large maximum amplitudes, such as above the ET.

With the impulsive period $q$ chosen as a variable parameter and with all the others fixed, a typical HE solution of the model is shown in Fig. 3(A) which is globally stable with $f_{2}=-0.0076<0$, where we observe how the parasitoid population $P_{n}$ oscillates in a stable cycle, and, in contrast, the pest $H_{n}$ rapidly decreases to zero. Fig. 3(B) is a HPP solution in which the host and parasitoid populations are persistent and the maximum amplitude of the host is relatively small (below the given ET if it is larger than 1). Fig. 3(C, D) are two HO solutions in which host populations have periodic outbreaks in (C) and irregular outbreaks (chaotic solution here) in (D).

Consequently, very interesting questions are (a) what is the stable region of the system? and (b) does the HE periodic solution lose its stability or not (local stability) when all parameters lie in the region $f_{2}>0$ ?

\subsection{Multiple attractors, coexistence and initial sensitivity}

The local stability of the HE solution and coexistence with other attractors including HPP and HO solutions are common properties of system (3.1) in the region $f_{2}>0$. Once the parameter set lies in the region of $f_{2}>0$, there are several types of coexistence including HE and HHP coexistence, HE, HHP and $\mathrm{HO}$ coexistence, which implies that $\mathrm{HE}$ is locally stable rather than globally stable in the region $f_{2}>0$. To confirm this, we fix all other parameters as in Fig. 3(A) except $r$. It follows from the function of $f_{2}$ that there is a threshold value of $r$, denoted by $r_{c}$ (where $r_{c}=1.8015$ ) such that HE is globally stable if $r<r_{c}$, and is locally stable (numerically) if $r>r_{c}$. At the interval $r_{c}<r<2.625$ (approximately), HE and HHP solutions coexist. One example of four attractors (HE, HHP, and two HO solutions with different outbreak frequencies) coexist at $r=2.63$ as shown in Fig. 4, from which we can see that for the HE solution the pest population outbreaks in the first few generations and then goes to extinction very quickly; three attractors (three types of solutions shown in Fig. 4(A, B, C)) coexist at $r=2.67$; another four attractors (HE, two HHP solutions and HO) coexist at $r=2.73$ as shown in Fig. 5. If $r$ is increased further, more complex multiple attractors can coexist including irregular HO, HE and HHP solutions.

These results clarify that the dynamical behaviour of system (3.1) is dominated by globally or locally stable periodic solutions and their coexistence, and further imply that the control of insect pests may depend on the initial densities of host and parasitoid populations. Thus, the initial attraction regions of these stable attractors play a pivotal role in pest management.

To show this, four basins of attraction with respect to four different types of coexistence are given in Fig. 6. It follows from their structures that the solutions of system (3.1) with relatively small parasitoid initial conditions (from the Cyan zone) will 

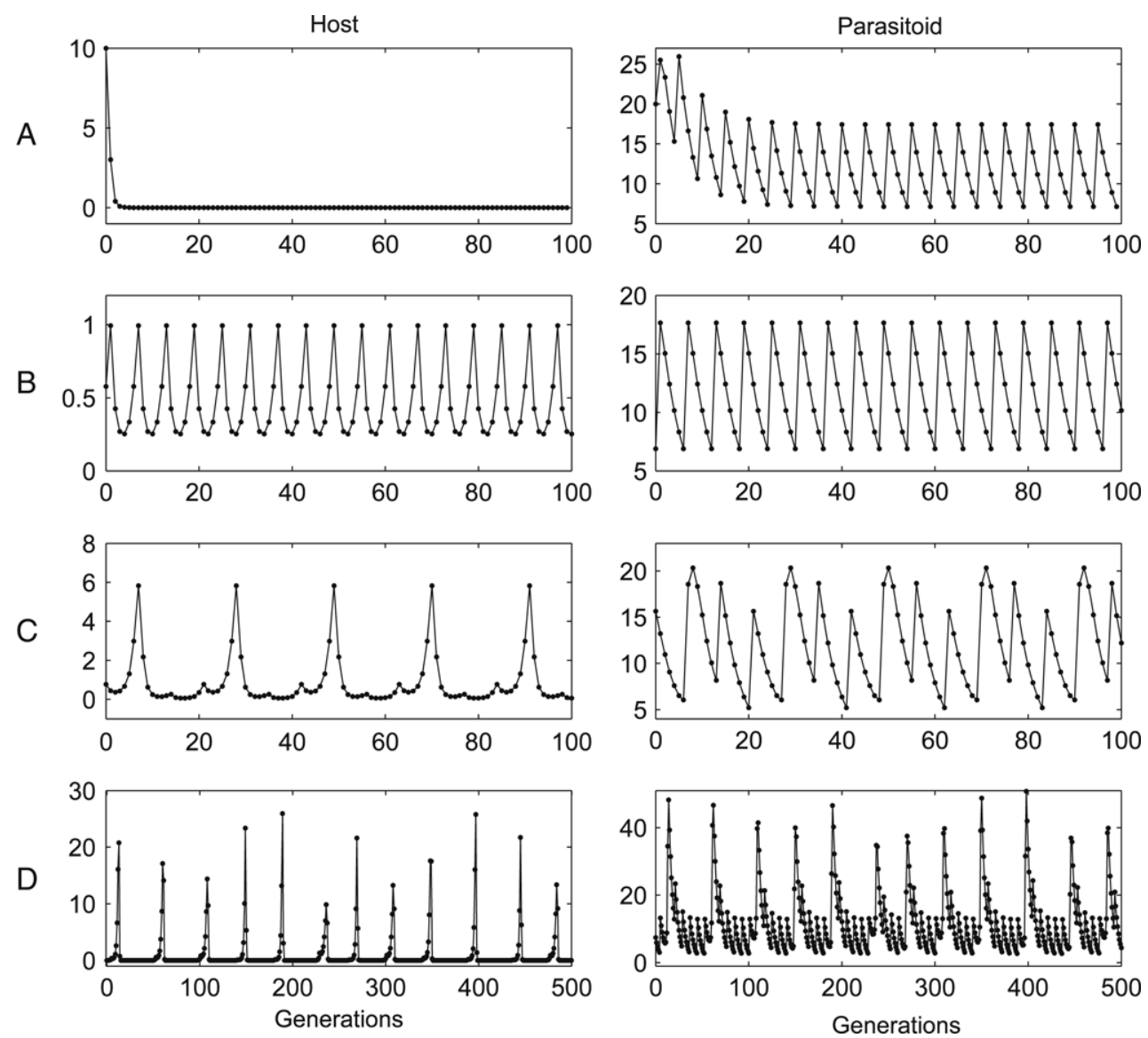

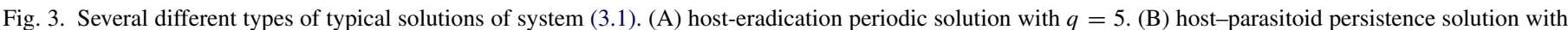

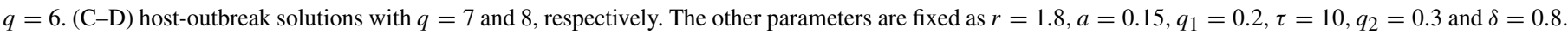

approach the HE solutions. However, from this region the first outbreak of the host population will be extremely high due to the low numbers of parasitoids and the exponential growth of the host population. Thus, this is not a biologically desirable solution and a large number of natural enemies would have to be released at this stage. With slightly increasing initial density of parasitoids, the structure of the basin attractor shown in Fig. 6(A) implies that all solutions which start from the Green zone will approach the HPP solutions which always oscillate below some threshold values (here ET) and are biologically desirable solutions. Moreover, it follows from bifurcation analysis (not shown here) that the HE and HPP solutions can coexist in a wide range of parameter space such as $r_{c}<r<2.625$ (approximately). But how can we implement an integrated management strategy such that the density of the host population does not exceed the given ET? To answer this question is one of our main aims in the next section.

With a slightly increasing initial density of the parasitoid, the structure of the basin attractors shown in Fig. 6(B-D) clarify that the small perturbation of initial conditions can significantly change the final state of the host population. This means that the insect pests may change their state from high density to low density and outbreak frequency or vice versa. Can this type of switch behaviour help us to control an insect pest? For example, can various dosages of insecticide application or different numbers of parasitoids released cause attractor transitions among these attractors?

\subsection{Different dosages of insecticide application and numbers of parasitoids released are crucial for controlling insect pests}

In order to avoid insecticide resistance, resistance strategies most often involve either mixing and applying pesticides together or alternating the use of available pesticides so that each generation of the pest is exposed to a compound with a different mode of action. But can such variations in doses and types of insecticides used and the numbers of natural enemies introduced affect the dynamics? Different numbers of natural enemies released and various dosages of pesticide applications or different pesticide applications can be mathematically expressed in terms of two parameters, $q_{1}$ and $q_{2}$, in model (3.1). That is, random perturbations due to variations in the dosages applied or releases (migration) of natural enemies can be taken into account with these two additional parameters, i.e. we have the following modified model:

$$
\left\{\begin{array}{l}
H_{n+1}=H_{n} \exp \left[r-a P_{n}\right], \\
P_{n+1}=H_{n}\left[1-\exp \left(-a P_{n}\right)\right]+\delta P_{n},
\end{array}\right\} \quad n=0,1,2, \ldots,
$$



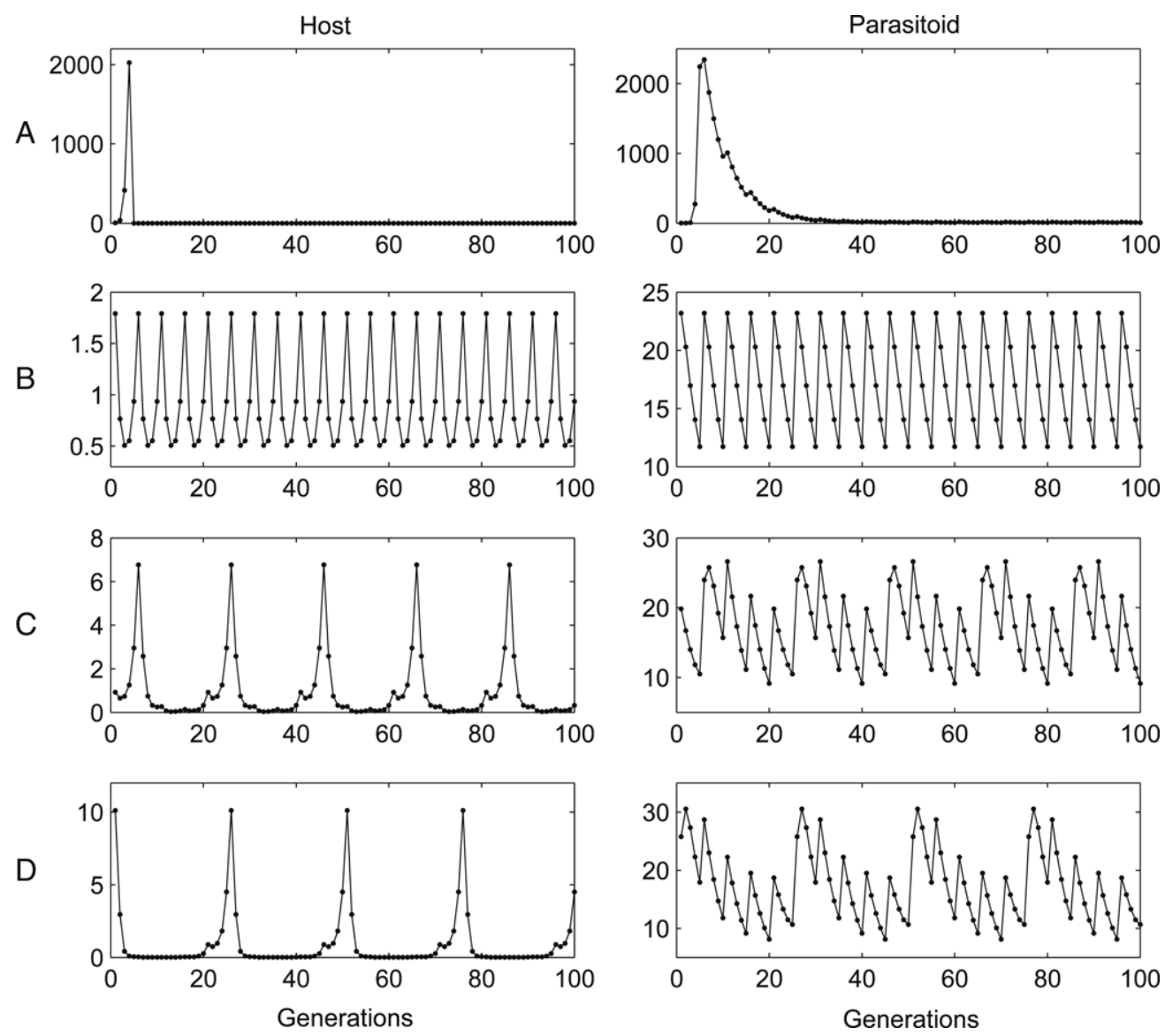

Fig. 4. Four coexisting attractors with $r=2.63, a=0.15, q_{1}=0.2, \tau=10, q=5, q_{2}=0.3$ and $\delta=0.8$. The initial conditions from top to bottom are $\left(H_{0}, P_{0}\right)=(3,1),(2.24,10.14),(0.92,19.8)$ and $(10.1,25.75)$, respectively. The periods are $5,5,20$ and 25 , respectively.

where $q_{1 \eta}=q_{1}+\eta_{1} u, q_{2 \eta}=q_{2}+\eta_{2} u$ and $u$ is a random variable uniformly distributed on $[-1,1]$ and $\eta_{1}, \eta_{2}>0$ represent the intensity of noise. One view of this noise is that it represents small random events of spraying, augmentation, immigration and mortality.

To understand how these small random perturbations affect the final state of the host population, we numerically investigated system (3.11) with respect to the switch-like transitions among the attractors shown in Fig. 4. That is, we asked do these stable attractors switch from one attractor to another once small random perturbations have been introduced? As an example, with all other parameter values fixed as in Fig. 4, it has been shown that there are four stable attractors which can coexist. If we choose the initial condition $\left(H_{0}, P_{0}\right)=$ $(10,25.47)$ (or $(10.76,23.24))$, the stable attractor without random perturbation is the pest-outbreak solution with larger amplitude (i.e. Fig. 4(D)). When we take into account the small random perturbations, numerical simulations imply that this solution can switch to another pest-outbreak solution with smaller amplitude (Fig. 4(C)) or switch to an HPP solution (Fig. 4(B)) at a random generation, as shown in Fig. 7. However, extensive numerical simulations indicate that HPP solutions are robust and are not affected by these types of small random perturbations. These numerical results confirm that different doses of insecticide application and natural enemies can influence the dynamics of the classical Nicholson-Bailey host-parasite system, and different numbers of parasitoids released may play key roles in insect pest control.

\subsection{The effects of insecticides on parasitoid and pest resistance}

It is well known that natural enemies are generally more adversely affected by chemical insecticides than the target pest. Because predators and parasitoids must search for their prey, they are generally very mobile and spend a considerable amount of time moving across plant tissue. This increases the likelihood that they will contact the insecticide. When insecticide timing also leads to the death of parasitoids, four different cases have been investigated according to the timing of application by Waage and Hassell (1982), Waage et al. (1985).

By using the same method as above, let us take the simplest case where in each impulsive point $q k$ there is an insecticide application that kills a constant fraction of hosts and which, in addition, may or may not affect the parasitoids. It is thus possible to rank in terms of their dynamic effects the different patterns of insecticide application in relation to the timing of parasitism. In system (3.1), we assume that an insecticide application does not affect the parasitoids. If the insecticide also affects parasitoids, then the following two possibilities will be considered according to the timing of application: 

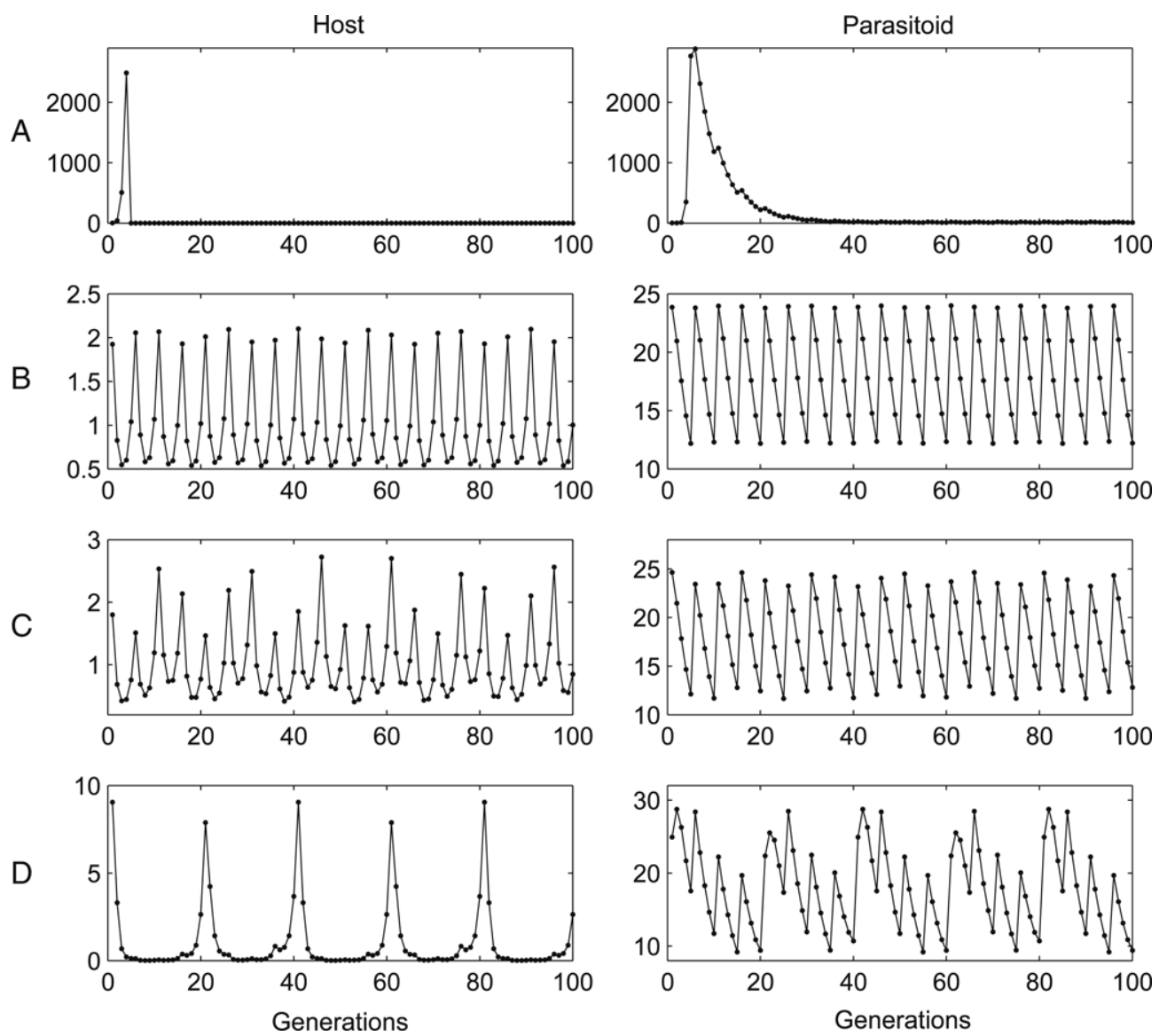

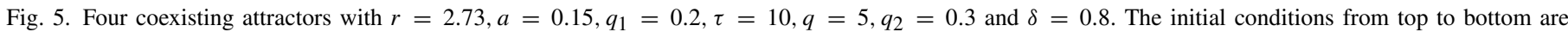
$\left(H_{0}, P_{0}\right)=(3,2),(1.92,23.85),(1.79,24.63)$, and $(9.04,24.92)$, respectively.

Case 1: Insecticides act prior to the releasing of the parasitoid, i.e.

$$
P_{q k^{+}}=\left(1+q_{2}\right)\left(1-q_{1}\right) P_{q k}+\tau .
$$

It follows that we have the following pest-eradication periodic solution

$$
\begin{aligned}
\left(0, P_{n}^{*}\right)= & \left(0, \frac{\tau \delta^{n-q s}}{1-\left(1+q_{2}\right)\left(1-q_{1}\right) \delta^{q}}\right), \\
& n \in\left[q s^{+}, q(s+1)\right) .
\end{aligned}
$$

For this case the condition which guarantees the existence of a pest-eradication periodic solution becomes:

$f_{3} \doteq 1-\left(1+q_{2}\right)\left(1-q_{1}\right) \delta^{q}>0$

and the globally stable condition becomes:

$$
\begin{aligned}
f_{4} \doteq & q r-\ln \left(\frac{1}{1-q_{1}}\right) \\
& -\frac{a \tau\left(1-\delta^{q}\right)}{\left[1-\left(1+q_{2}\right)\left(1-q_{1}\right) \delta^{q}\right](1-\delta)}<0 .
\end{aligned}
$$

Case 2: Insecticides act after the releasing of the parasitoid, i.e.

$P_{q k^{+}}=\left(1-q_{1}\right)\left[\left(1+q_{2}\right) P_{q k}+\tau\right]$.
It follows that we have the following pest-eradication periodic solution

$\left(0, P_{n}^{*}\right)=\left(0, \frac{\left(1-q_{1}\right) \tau \delta^{n-q s}}{1-\left(1+q_{2}\right)\left(1-q_{1}\right) \delta^{q}}\right)$,

$n \in\left[q s^{+}, q(s+1)\right)$.

The condition which guarantees the existence of this solution is the same as Case 1, but the globally stable condition becomes:

$$
\begin{aligned}
f_{5} \doteq & q r-\ln \left(\frac{1}{1-q_{1}}\right) \\
& -\frac{a \tau\left(1-q_{1}\right)\left(1-\delta^{q}\right)}{\left[1-\left(1+q_{2}\right)\left(1-q_{1}\right) \delta^{q}\right](1-\delta)}<0 .
\end{aligned}
$$

In order to investigate the effect of insecticide on the stable region of the HE solution when it also affects the parasitoid, we fix all parameters as in Fig. 3(A) except $q$ and $q_{1}$.

It follows from Fig. 8 that the globally stable region of the HE periodic solution has been extremely reduced if the insecticide also affects the parasitoids. These results clarify that when insecticide timing also leads to the death of parasitoids, pest depression is reduced and pest resurgence may or may not appear, depending again on the timing of the application. However, the advantage of the modelling methods presented here is that we can avoid this practical problem by choosing different timings for the insecticide applications and 
A

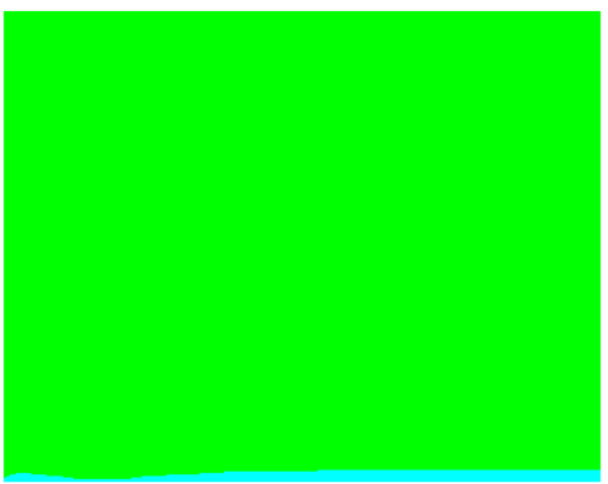

C

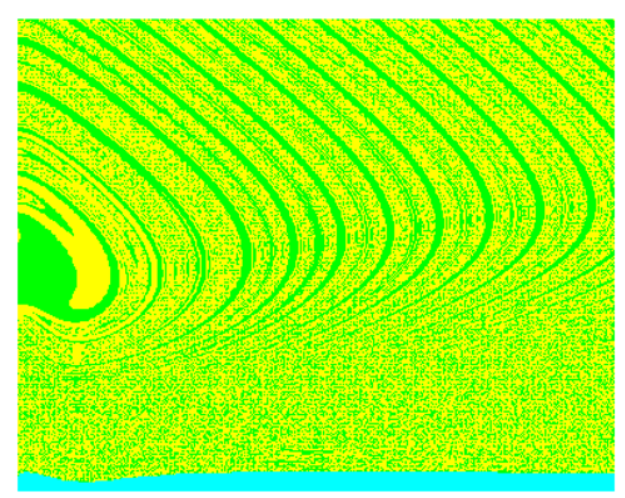

B

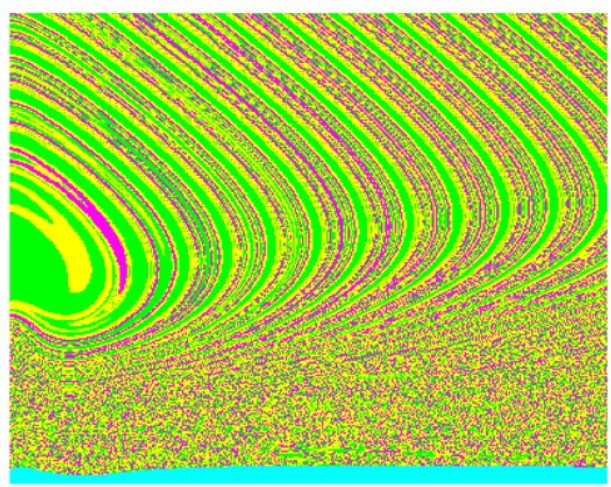

D

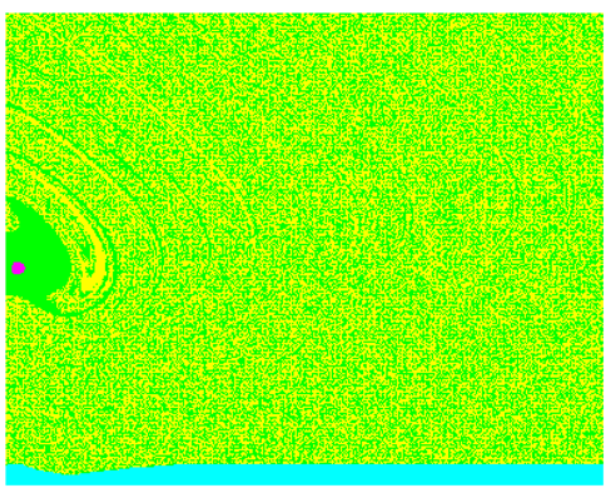

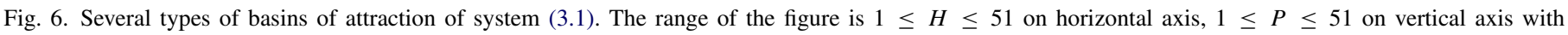

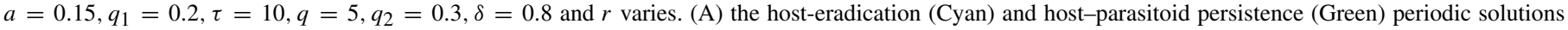

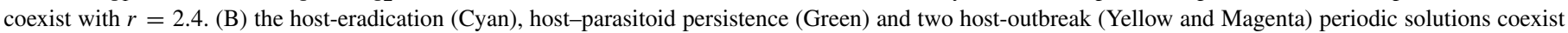

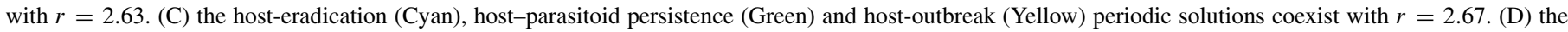

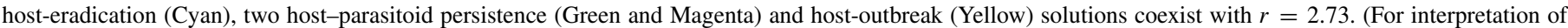
the references to colour in this figure legend, the reader is referred to the web version of this article.)

the parasitoid releases. For example, we can spray insecticide before or after releasing natural enemies in $m$ generations (where $m<q$ ), i.e. the third and fourth equations in system (3.1) are replaced by the following two equations:

$\left\{\begin{array}{l}H_{q k \pm m}^{+}=\left(1-q_{1}\right) H_{q k \pm m}, \\ P_{q k}^{+}=\left(1+q_{2}\right) P_{q k}+\tau,\end{array} \quad k=1,2, \ldots\right.$.

These changes do not affect the existence and stability of the HE periodic solution.

As noted in the introduction, pesticide resistance is a genetically-based phenomenon. Resistance develops when an insect pest is exposed to a pesticide and not all of the insects are killed. Those individuals that survive have often done so because they are genetically predisposed to be resistant to the pesticide. Repeated applications and higher rates of the insecticide will kill increasing numbers of individuals, but some resistant insects will survive. The offspring of these survivors will carry the genetic make-up of their parents. These offspring, many of which will inherit the ability to survive the exposure to the insecticide, will increase in proportion with each succeeding generation. The same method used by Tang et al. (2005) can be employed to investigate the effect of insecticide resistance on the stability of the HE periodic solution.
In a real world, complete eradication of pest populations is generally not possible, nor is it biologically or economically desirable. A good pest control programme should reduce pest populations to levels acceptable to the public. However, how can we estimate and predict the dosage and frequency of applications of insecticide and releases of natural enemies once the density of host population reaches the given ET? This is the question we will address in the following section.

\section{Host-parasitoid models with economic threshold}

So far, the host-parasitoid model with a fixed periodic control strategy was investigated and the results obtained here imply that the pest population can be completely eradicated under suitable conditions. However, the disadvantage of models (3.1) or (3.11) is that an integrated pest management strategy is applied whether the density of host exceeds the ET or not, which is not cost-effective. Consequently, the frequency of insecticide application is relatively high and, combined with the use of high dosage rates, detrimental impacts on natural enemies and on the environment will be more severe than if control is only applied when the ET has been reached.

As noted in the introduction, an IPM control, including biological control (augmenting natural enemies), cultural control (alternate hosts, crop rotation, etc) and physical and 
A
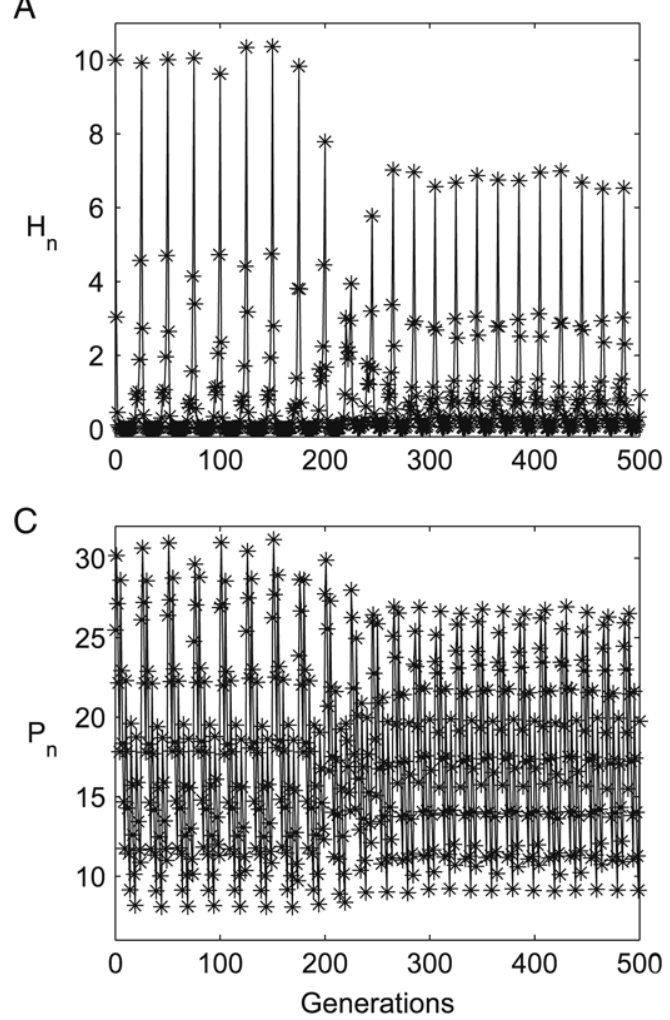

B

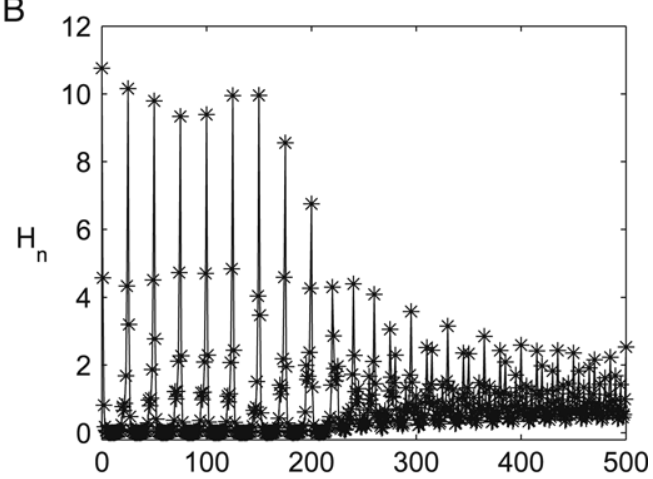

D

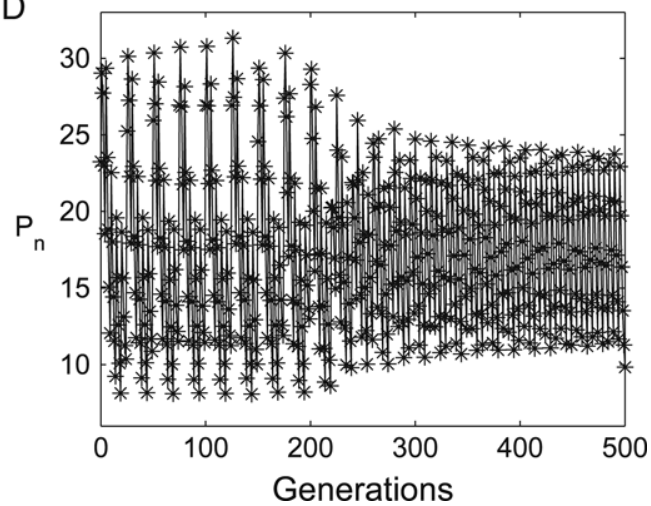

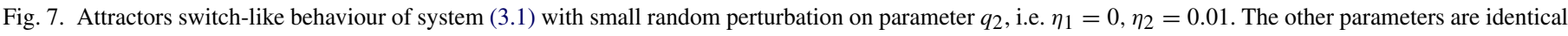

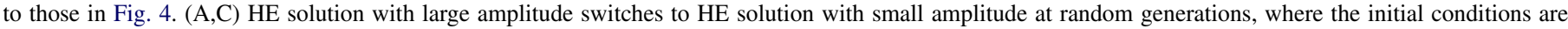

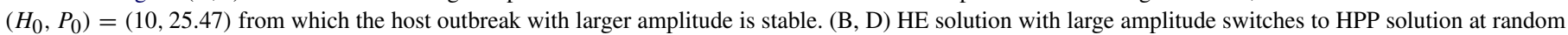
generations, where the initial conditions are $\left(H_{0}, P_{0}\right)=(10.76,23.24)$ from which the host outbreak with larger amplitude is stable.

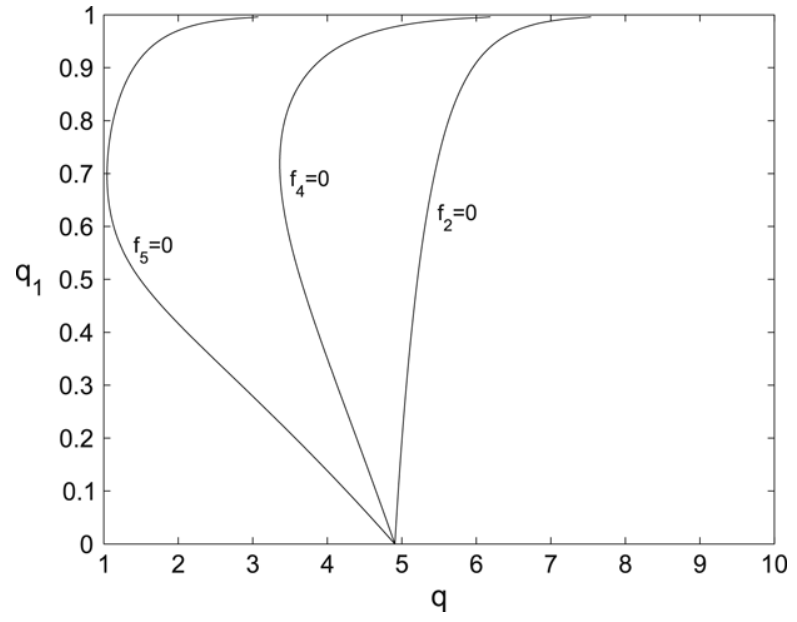

Fig. 8. The $q-q_{1}$ parameter plane shows the effects on the globally stable region of the HE periodic solution when an insecticide application may or may not affect the parasitoids. The threshold curves of three functions $f_{2}, f_{4}, f_{5}$ are plotted against parameters $q$ and $q_{1}$ which determine the global stability of the host-eradication periodic solution, i.e. the right sides of the three curves are the globally stable region of the corresponding HE periodic solutions if they exist. The other parameters are $r=1.8, a=0.15, \tau=10$ and $q_{2}=0.3$.

mechanical control, only needs to be applied when the pest population reaches the ET. However, the solutions of discrete models do not exactly go through ET due to the discontinuous and complexity of their solutions. Usually, the solutions may suddenly jump above or below ET at some generations. If this happens, the control tactics including biological control and cultural control are usually incapable of reducing the density of the host population below the ET immediately due to the response delay. Thus, an insecticide application is needed at this time because insecticides are easy to apply, fast-acting, and in most instances can be relied on to control the pests.

For example, if the insect pest has an outbreak and exceeds the ET at generation $k(k>0)$, i.e. $H_{k} \exp \left[r-a P_{k}\right]>E T$ (Mathematically, we assume the initial density of host $H_{0}$ is less than ET. Otherwise, the initial density of the host is chosen after an application of IPM strategy, i.e. $H_{0^{+}}=H_{0}$ ). In this case, an insecticide application needs to be applied and at least a number of $q_{1 k} H_{k} \exp \left[r-a P_{k}\right]$ insect pests should be killed such that $\left(1-q_{1 k}\right) H_{k} \exp \left[r-a P_{k}\right]=E T$, where $1-q_{1 k}$ is the proportionate survival rate after an insecticide application which now depends on the densities of the host and parasitoid populations. In order to control it more effectively, other control tactics including biological control are applied at the same generation. Thus, we have the following new model:

$$
\left\{\begin{array}{l}
H_{n+1}=\min \left\{H_{n} \exp \left[r-a P_{n}\right], E T\right\}, \\
P_{n+1}=H_{n}\left[1-\exp \left(-a P_{n}\right)\right]+\delta P_{n}, \\
P_{n+1^{+}}=\left(1+q_{2}\right) P_{n+1}+\tau, \quad \text { if } H_{n+1}=E T, \\
H_{0^{+}}=H_{0}<E T, P_{0^{+}}=P_{0}
\end{array}\right.
$$

where $n=0,1,2, \ldots, E T$ denotes the economic threshold (pest population density at which the control measures should be determined to prevent an increasing pest population from 

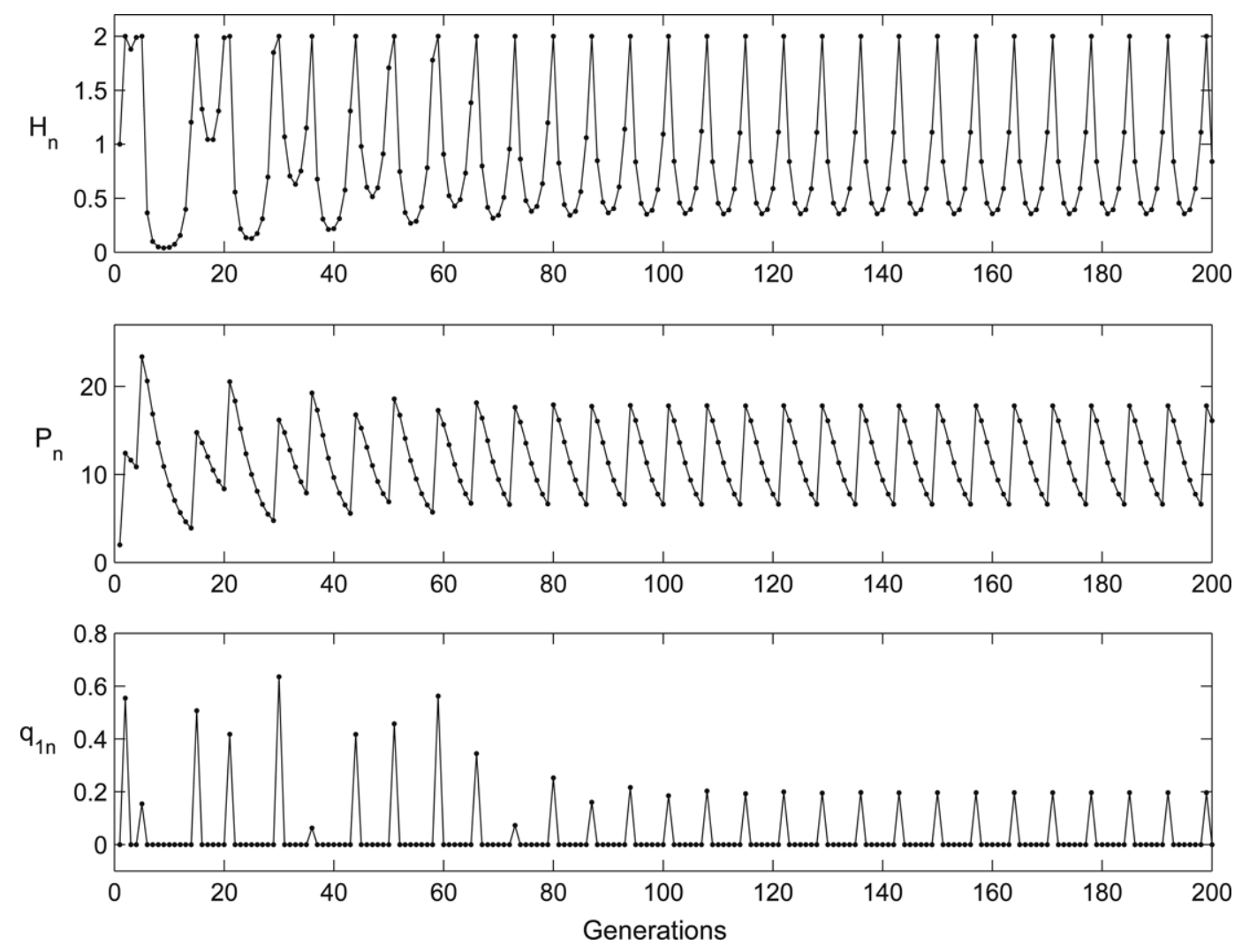

Fig. 9. Periodic solution of system (4.1) with $E T=2, r=1.8, a=0.15, q_{2}=0.3, \tau=10$ and $\delta=0.8$. The initial conditions are $\left(H_{0}, P_{0}\right)=(1,2)$.

reaching the economic injury level). What we want to do is design a control strategy such that the pest population lies below ET through model (4.1). That is, to determine when we should implement an IPM strategy and how many insect pests should be killed which is equivalent to calculating

$q_{1 n}= \begin{cases}1-\frac{E T}{H_{n} \exp \left(r-a P_{n}\right)}, & \text { if } H_{n} \exp \left[r-a P_{n}\right]>E T, \\ 0, & \text { otherwise. }\end{cases}$

In order to compare the results of this model with the pesteradication periodic solution shown in Fig. 3(A), we fix the parameters as $r=1.8, a=0.15, \delta=0.8, \tau=$ $10, q_{2}=0.3$, and assume that the economic threshold is relative small, i.e. $E T=2$. In what follows, we try to numerically find the periodic solution of system (4.1) such that the maximum amplitude of the host population does not exceed ET. Consequently, the outbreak frequency (impulsive frequency) of the host and time series of $q_{1 n}$ are determined which play the key roles in the design of an IPM strategy. The results shown in Fig. 9 indicate that there does exist a periodic solution for the system (4.1), as expected. It follows from Fig. 9 that the host and parasitoid populations oscillate periodically and the maximum amplitude of the host population is no larger than ET. The most interesting results here are that the period of this periodic solution is 7 and $q_{1 n}$ stabilizes to a constant $q_{1}$ (here $q_{1}=0.1963$ ) (in Fig. 3(A), the period of the HE solution is $5(q=5)$ and the proportionate killing rate is $\left.0.2\left(q_{1}=0.2\right)\right)$. This confirms that the frequency of insecticide application, host outbreak and spraying dosages are significantly reduced if we aim to keep the host populations below ET and avoid ecological damage rather than eradicate them.

The effect of the host intrinsic growth rate $r$ on host mean outbreak period and the mean instantaneous killing rate of $q_{1 n}$ can be calculated from the model (4.1) numerically (Fig. 10). Mean outbreak period is the period of the host cycle averaged over several periods. Mean instantaneous killing rate of $q_{1 n}$ is the mean value of the positive values of $q_{1 n}$ over several generations (200 generations here). The model predicts that the mean outbreak period of the host population decreases as a function of $r$, i.e. high intrinsic growth rates are associated with frequent outbreaks, while the mean outbreak period of the host population increases as a function of ET. However, the mean fraction of the instantaneous killing rate of $q_{1 n}$ only depends on the corresponding solution as well as its initial condition. Therefore, the use of economic thresholds, field monitoring, and record keeping for both pest and parasitoid by date are important parts of an IPM programme. These dates are important for making management decisions during a season and will give important historical perspective to the effectiveness of any controls implemented. Here, the time series $q_{1 n}$ provides useful information on this aspect including on the timing of an IPM application and the host-killing rate.

However, we note that the dynamical behaviour of system (4.1) with unfixed impulsive moment is much more complex than that of system (3.1) with fixed moment. It is noted that the dynamical behaviour of system (3.1) is dominated by several types of periodic solutions and their coexistence, even with very 

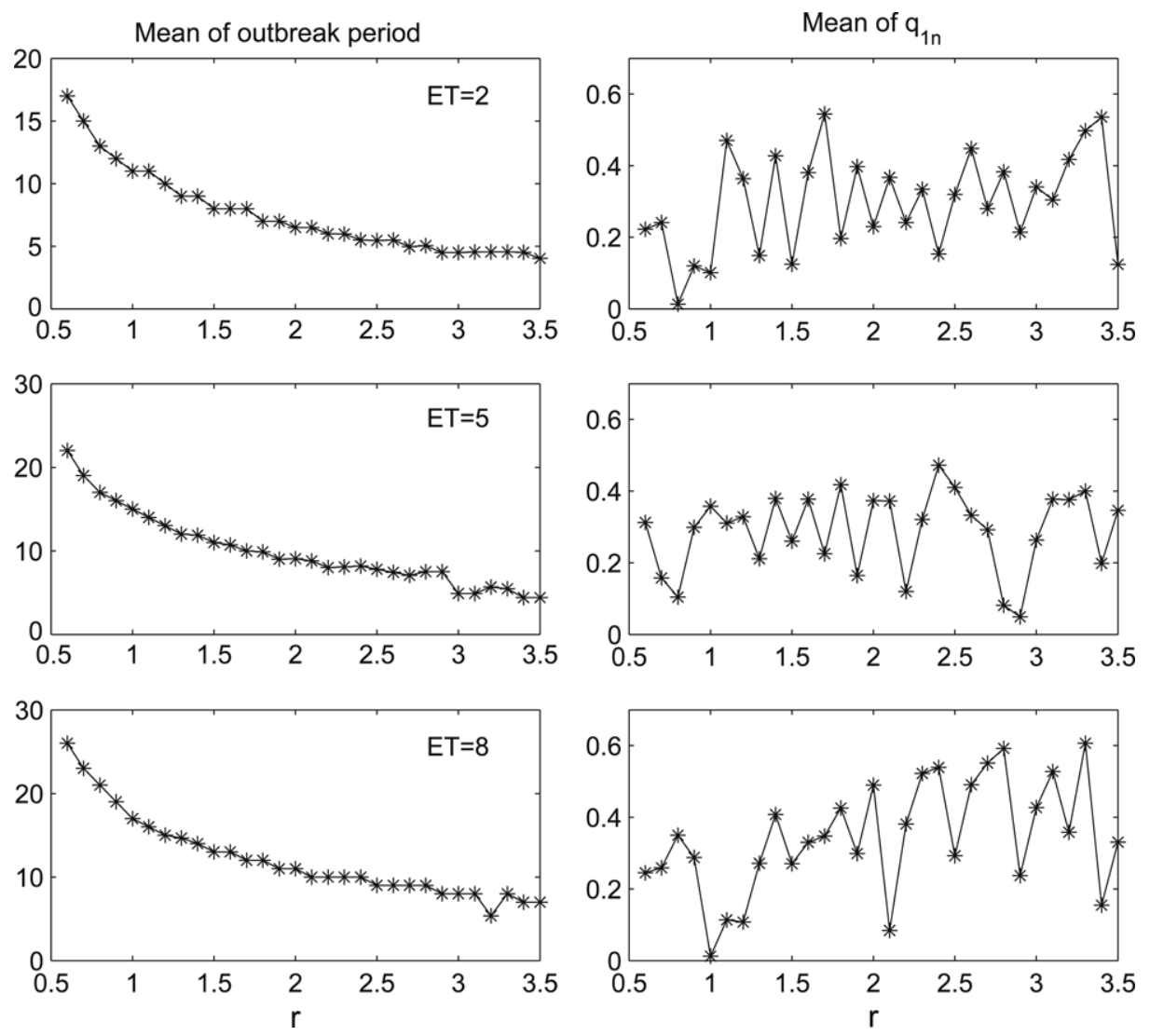

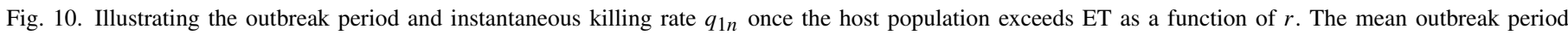

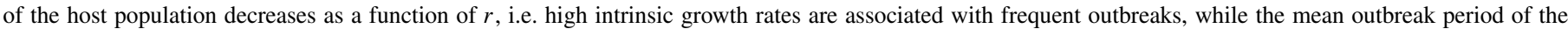

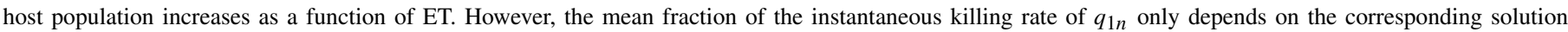

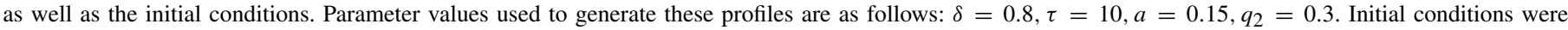

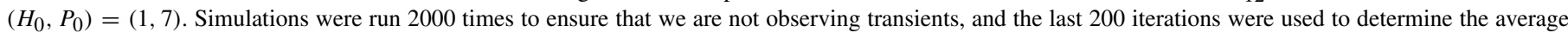
outbreak period and the mean killing proportion of $q_{1 n}$.

high intrinsic growth rates. While it follows from bifurcation analysis (Fig. 11) that the dynamical behaviour of system (4.1) is very complex, including period doubling bifurcation and chaotic solutions, even with very small intrinsic growth rates. In particular, the complex pattern in Fig. 11(C) shows that the proportionate killing rate of $q_{1 n}$ is difficult to estimate and to predict. These results indicate that, in practice, it is a very difficult task to control the host population such that its density does not exceed ET.

Meanwhile, system (4.1) also has a certain type of coexistence if the intrinsic growth rate of the host population is large enough. Figs. 12 and 13 show two of examples of attractor coexistence of system (4.1) and their corresponding basins of attractions are given in Fig. 14.

\section{Biological conclusions}

When using integrated pest management as an approach to control insect pests one must be committed to a long term strategy. Regular field monitoring must be done to keep track of both pest and beneficial insect populations for an IPM programme to be effective. Proper identification of insect pests and a basic knowledge of economic thresholds are essential for an IPM programme to be successful. To properly estimate and predict the timing of an IPM application and the dosage of insecticide application, we have extended the classical Nicholson-Bailey model to include an IPM control strategy. According to different control purposes, eradication of an insect pest or keeping its density below ET, two new modelling methods are presented in this paper.

For the first model, we have been able to show that the HE periodic solution is globally stable if the intrinsic growth rate of the host population is less than the summation of the mean host-killing rate and the mean parasitization rate during the impulsive period, which means that any single control strategy (biological control only or chemical control only) will significantly reduce the stability region of the $\mathrm{HE}$ periodic solution. This is consistent with observation and experience that any single method approach to pest control is generally neither possible nor desirable over the long term.

The sufficient condition which guarantees the global stability of the HE solution can help us to design the control strategy. For example, we can at least numerically calculate the impulsive period $q$ from equation $f_{2}=0$ once all other parameters are given, which plays an important role in decision-making for an IPM strategy. It can also be used 

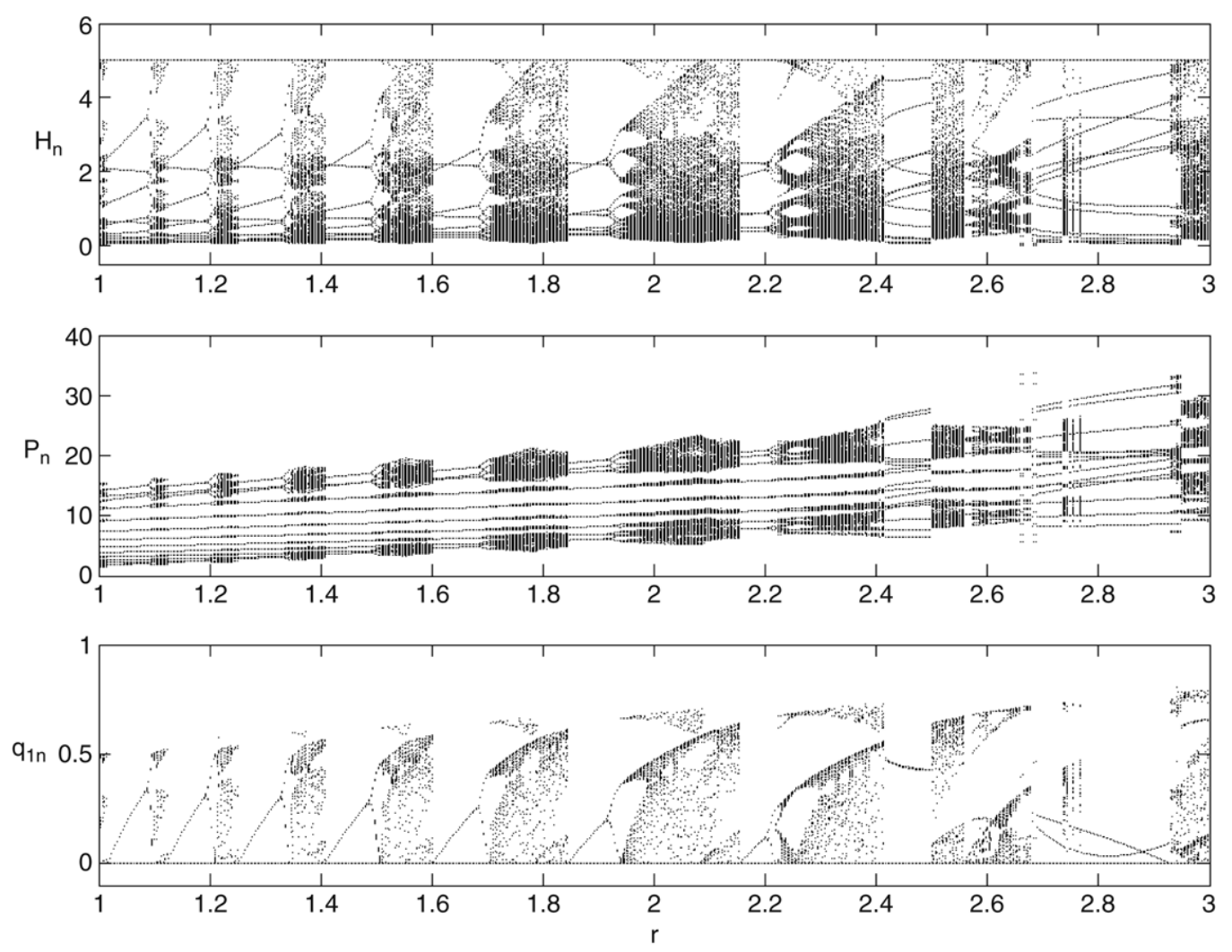

Fig. 11. Bifurcation diagram of system (4.1) with $E T=5, a=0.15, q_{2}=0.3, \tau=10$ and $\delta=0.8$. The initial conditions are $\left(H_{0}, P_{0}\right)=(1,18)$.

to estimate the host-killing rate $q_{1}$ or parasitoid release rate $q_{2}$. The stable conditions of HE solutions also indicate that the parasitoid intergenerational survival rate plays a key role in maintaining the periodic oscillations of parasitoid species. Therefore, conservation and management measures to increase the parasitoid's reproductive rate, mobility and overwintering survival rate are of great importance in insect pest control. As natural enemies are generally well adapted to their local environments and to the target pest, their conservation is generally simple and cost-effective.

Interestingly, the HE periodic solution does not lose its stability when the intrinsic growth rate of the host population is larger than the summation of the mean host-killing rate and mean parasitization rate, but it coexists with several other stable attractors including HPP and HO solutions. This implies that the control of an insect pest depends on the initial densities of host and parasitoid population. Moreover, the structure of basins of attraction indicates that small random perturbations may dramatically affect the final result of an IPM control strategy.

However, the stable HO solutions with large amplitudes can switch to the stable HO solution with relatively small amplitude or switch to the stable HPP solution at a random generation while the stable HPP solutions are robust to random perturbation. These results confirm that varying dosages of insecticide applications and numbers of parasitoids released are crucial for insect control and pesticide resistance.

A good pest control programme should reduce pest populations to levels acceptable to the public rather than eradication. Our second type of model is proposed based on this ideal. Numerical investigations confirm that a periodic solution with maximum amplitude below ET does exist for the new model. Comparing the period and stable host-killing rate with those in the model with fixed moments, we see that the frequency of insecticide application and the dosage that needs to be applied are significantly reduced. Consequently, this may reduce damage to the environment and avoid pesticide resistance.

In reality, uncertainties or lack of information about pests, damage, controls and prices also exist. In other words, the type of control a farmer applies to his main crop influences his control decision for the second crop. Imperfect knowledge and limited skills concerning pest control decisions and the presence of natural disturbances may render actual decisions "inferior" to those predicted by the models. Importantly, the time series of $q_{1 n}$ which was calculated from system (4.1) provides important information including the timing of an IPM application and the host-killing rate, which are important factors in the design of appropriate IPM strategies and control decision-making.

However, the complexity of $q_{1 n}$ further confirms that an IPM strategy, in practice, is difficult to implement. The estimation of ET and EIL is generally a complex matter based on detailed operations of pest ecology as it relates to bioclimatology, predation, diseases, the effect of host-plant resistance and the environmental consequences of applied control interventions (Metcalf and Luckman, 1975). So a challenge for future research is to combine the decision-making models developed by Headley (1968) with the models developed in this paper. 

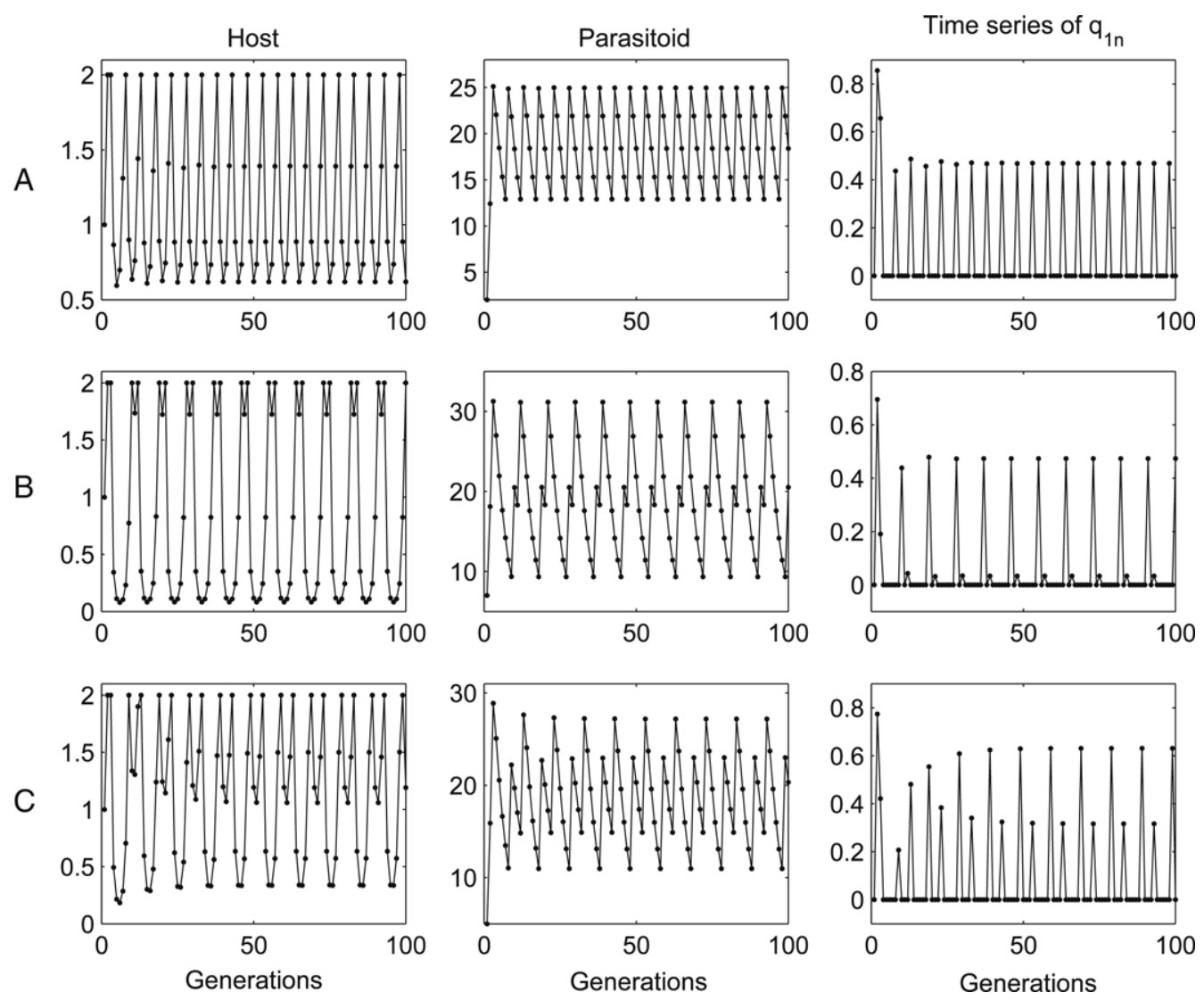

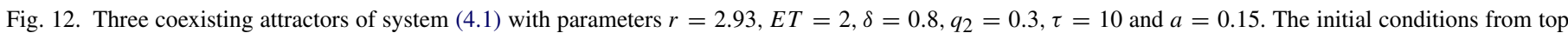

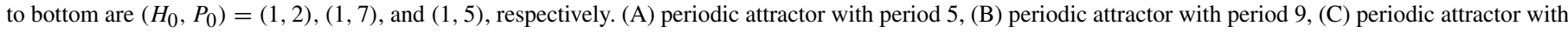
period 10 .

As far as practical applications are concerned, it is pest control systems in glasshouses for which there are most data. For instance the parasitoid E. formosa is now commercially available for controlling the whitefly Trialeurodes spp. in greenhouses and conservatories for which weekly releases are recommended. Similarly, the predatory mite $P$. persimilis is available for controlling spider mites Tetranychus spp., for which the inundative releases are advised, at least initially. In both instances initial reductions of the pests using pesticides may be appropriate to bring the pest populations down to levels manageable by the biological control agents. In such cases it is important that the insecticide residues, or residues from other pesticides used against other pests in the same arena, do not kill the introduced agents. Thus the timing of the introduction of the agents in relation to when the pesticide applications occurred is important in IPM. For instance, Udayagiri et al. (2000) investigated the survival of the parasitoid Anaphes iole at up to six different times after exposure to each of six insecticides, two acaricides and six fungicides and showed how the parasitoid releases and their timings could or could not be integrated with pesticide use.

Apart from timings of releases our models also consider variation in intrinsic rates of increase $(r)$, searching efficiencies (a) and density-independent survival $(\delta)$. The intrinsic rate of increase of $P$. persimilis may be as high as 0.4 per generation but this varies with prey type and the plant species involved
(McMurtry and Croft, 1997). There is also much variation in $a$ (here treated as the area of discovery of (Nicholson and Bailey, 1935)), which is itself density dependent decreasing with increasing parasitoid density (Hassell and Varley, 1969). A mean value of $0.18 \mathrm{~m}^{2}$ per generation has been estimated for $a$ for Cyzenis albicans parasitizing the winter moth Operophtera brumata (Hassell, 2000b) and that for Eurytoma tibialis parasitizing Urophora jaceana was $0.4 m^{2}$ in one year and $0.2 \mathrm{~m}^{2}$ in another (Varley et al., 1973). Outcomes in terms of percentages of hosts parasitized by biological control agents range from 3\% to 100\% (Hawkins et al., 1993) with obvious implications for the success or failure of the biological control programmes involved. Estimates of $\delta$ can be attributed to a variety of factors including immigration or emigration rates in field systems and release rates in glasshouses. Van Dreische and Lyon (2003) give examples of release rates (0.5, 1 and 3 female insects per plant per week), different release patterns (constant, front end loaded and back end loaded) and of economic thresholds (ET of 2 living nymphs or pupae per leaf) for Eretmocerus eremicus introductions against whitefly infesting commercial pointsettia plants Euphorbia pulcherrima, in combination with the use of an insect growth regulator pesticide. Success was achieved with release rates of the parasitoid of 0.5 per plant per week, combined with a midseason application of kinoprene at a cost of only US $\$ 0.10-0.14$ per plant protected, less than or equal to the cost of conventional 

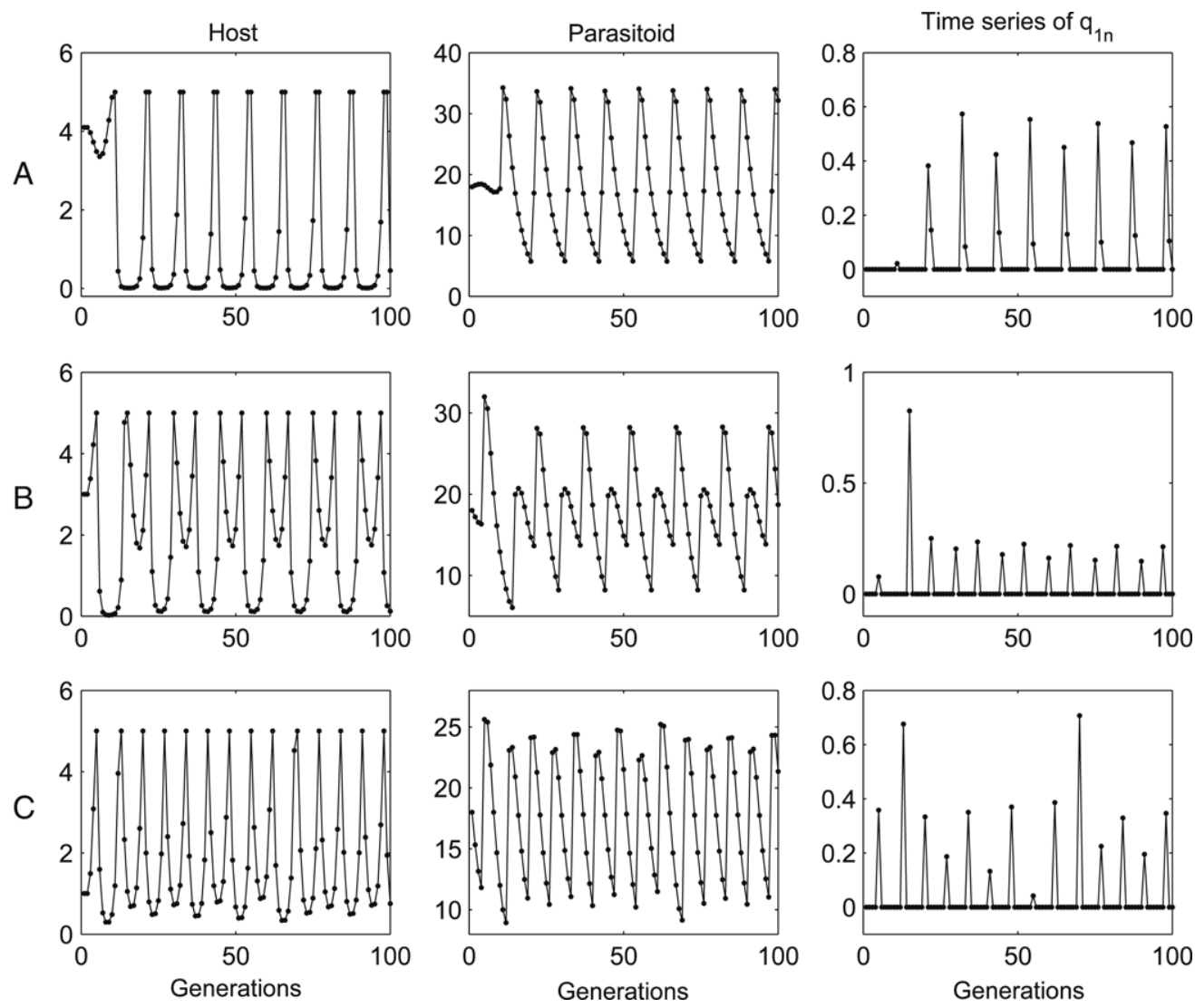

Fig. 13. Three coexisting attractors of system (4.1) with parameters $r=2.7, E T=5, \delta=0.8, q_{2}=0.3, \tau=10$ and $a=0.15$. The initial conditions from top to bottom are $\left(H_{0}, P_{0}\right)=(4.1,18),(3,18)$, and $(1,18)$, respectively. (A) periodic attractor with period $10,(\mathrm{~B})$ periodic attractor with period 15 , (C) chaotic attractor.

A

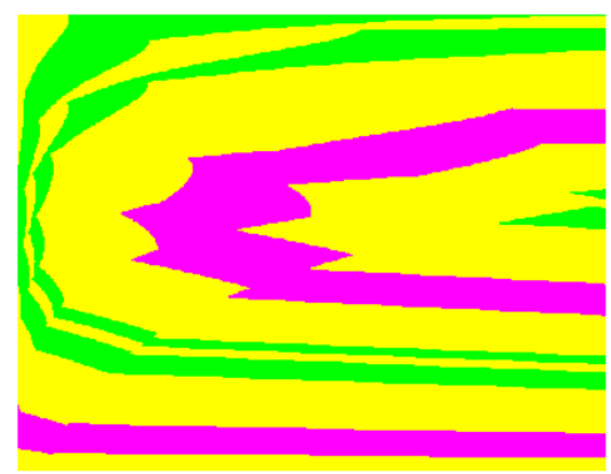

B

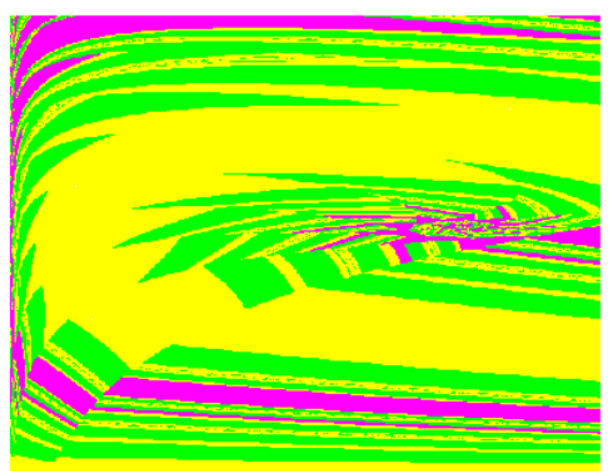

Fig. 14. Two basins of attraction of system (4.1) corresponding to Figs. 11 and 12 . The range of (A) is $0.01 \leq H \leq 2$ on horizontal axis, $0.1 \leq P \leq 34$ on vertical axis. The range of (B) is $0.01 \leq H \leq 5$ on horizontal axis, $0.1 \leq P \leq 34$ on vertical axis. Parameters are as follows: $a=0.15, \tau=10, q_{2}=0.3, \delta=0.8$. $r=2.93, E T=2$ in (A) and $r=2.7, E T=5$ in (B). The magenta, green and yellow points are attracted to the attractors shown in Figs. 11 and 12 from top to bottom, respectively. (For interpretation of the references to colour in this figure legend, the reader is referred to the web version of this article.)

methods. Given the extensive variation in parameter values in such real systems, exploration with models using a wide range of each, as described here, is appropriate and allows flexibility in predicting outcomes for any given case for which the adequate empirical data are available.

Extensive numerical bifurcation analysis confirmed that the periodicity and coexistence of model (3.1) (Figs. 3-5) and the complex behaviour of model (4.1) (Fig. 11) are common to both models and these properties can exist for a wide range of parameters. Thus, the choice of which parameters to vary, and what values to use is unimportant, as many others would give similar results and reproduce most of the behaviour shown in this paper, within finite generations for realistic parameter values.

The majority of this work is based on deterministic models of the Nicholson-Bailey host-parasitoid interaction with pulse perturbations. This raises the interesting question of how robust the models are to various forms of environmental and demographic stochasticity, as it is well known that stochasticity can significantly affect the persistence and 
dynamics of populations (Bonsall and Hastings, 2004). The switch-like behaviour on small random perturbations confirms that the environmental stochasticity has little effect on the coexistence of attractors. In particular, the HPP solutions are robust to environmental stochasticity. However, demographic noise may affect the population dynamics more broadly, and is most influential in small populations. Stochastic fluctuations at small population sizes tend to be amplified by the dynamics to cause massive population variability, i.e. demographic stochasticity has a destabilizing effect. How do environmental and demographic stochasticity affect the rich dynamic behaviour described in this paper and influence the IPM strategy, including coexistence and the structure of the basin attractors? The introduction of stochasticity also allows us to investigate the interesting question of whether stochasticity is beneficial to IPM strategies or not, a question for future research directions.

This work focused entirely on the simplest Nicholson-Bailey model with impulsive effects and the temporal interactions of an insect host and its parasitoid. A number of stabilizing factors such as spatial heterogeneity, density-dependent growth of the host and functional responses of the parasitoid will be considered in future and will be reported elsewhere.

\section{Appendix}

The Proof of Theorem 3.1. It follows from $q<\frac{1}{r} \ln \left(\frac{1}{1-q_{1}}\right)+$ $\frac{a \tau\left(1-\delta^{q}\right)}{r\left(1-\left(1+q_{2}\right) \delta^{q}\right)(1-\delta)}$ that we have $\left(1-q_{1}\right) \prod_{n=q s}^{q(s+1)-1}[\exp (r-$ $\left.\left.a P_{n}^{*}\right)\right]<1$. Therefore there exists $\epsilon>0$ sufficiently small such that

$\delta_{1} \doteq(1-p) \prod_{n=q s}^{q(s+1)-1}\left[\exp \left(r-a\left(P_{n}^{*}-\epsilon\right)\right)\right]<1$.

Note that $P_{n+1}>\delta P_{n}$, and consider the following impulsive difference equation:

$$
\left\{\begin{array}{l}
Q_{n+1}=\delta Q_{n}, \quad n=0,1,2, \ldots, \\
Q_{q k^{+}}=\left(1+q_{2}\right) Q_{q k}+\tau, k=1,2, \ldots
\end{array}\right.
$$

It follows from Lemma 3.1 that we have $P_{n} \geq Q_{n}$ and $Q_{n} \rightarrow P_{n}^{*}$ as $n \rightarrow \infty$. Hence

$P_{n} \geq Q_{n}>P_{n}^{*}-\epsilon$

for all large $n$. For simplification we may assume that (A3) holds for all $n \geq 0$. From (3.1) we get

$$
\left\{\begin{array}{l}
H_{n+1} \leq H_{n} \exp \left(r-a\left(P_{n}^{*}-\epsilon\right)\right), \quad n \geq 0, \\
H_{q k^{+}}=\left(1-q_{1}\right) H_{q k}, \quad k \geq 1 .
\end{array}\right.
$$

It follows that we have

$$
\begin{aligned}
H_{q(s+1)} & \leq H_{q s^{+}} \prod_{n=q s}^{q(s+1)-1} \exp \left(r-a\left(P_{n}^{*}-\epsilon\right)\right) \\
& =H_{q s}\left(1-q_{1}\right) \prod_{n=q s}^{q(s+1)-1} \exp \left(r-a\left(P_{n}^{*}-\epsilon\right)\right) \\
& =H_{q s} \delta_{1} .
\end{aligned}
$$

It follows from (A1) that $H_{q s} \leq H_{0^{+}} \delta_{1}^{s}$ and $H_{q s} \rightarrow 0$ as $s \rightarrow \infty$. Therefore $H_{n} \rightarrow 0$ as $n \rightarrow \infty$ since $0<H_{n} \leq$ $H_{q s}\left(1-q_{1}\right) \exp (q r)$ for $n \in\left[q s^{+}, q(s+1)\right)$. This completes the proof.

\section{References}

Barclay, H.J., 1982. Models for pest control using predator release, habitat management and pesticide release in combination. J. Appl. Ecology 19, 337-348.

Barlow, N.D., Moller, H., Beggs, J.R., 1996. A model for the effect of Sphecophaga vesparum as a biological control agent of the common wasp in New Zealand. J. Appl. Ecology 33, 31-34.

Barron, M.C., Wratten, S.D., Barlow, N.D., 2004. Phenology and parasitism of the red admiral butterfly Bassaris gonerilla (Lepidoptera: Nymphalidae). New Zealand J. Ecology 28 (1), 105-111.

Beggs, J.R., Harris, R.J., Read, P.E.C., 1996. The invasion success of the wasp parasitoid Sphecophaga vesparum (Curtis) in New Zealand. New Zealand J. Zoology 23, 1-9.

Beddington, J.R., Free, C.A., Lawton, J.H., 1975. Dynamic complexity in predator-prey models framed in difference equations. Nature 255, 58-60.

Beddington, J.R., Free, C.A., Lawton, J.H., 1978. Characteristics of successful natural enemies in models of biological control of insect pests. Nature 273, 513-519.

Bonsall, M.B., Hastings, A., 2004. Demographic and environmental stochasticity in predator-prey metapopulation dynamics. J. Anim. Ecol. 73 , 1043-1055.

Broadhead, E., Cheke, R.A., 1975. Host spatial pattern, parasitoid interference and the modelling of the dynamics of Alaptus fusculus (Hym.:Mymaridae), a parasitoid of two Mesopsocus species (Psocoptera). J. Anim. Ecol. 44, 767-793.

Briggs, C.J., Borer, E.T., 2005. Why short-term experiments may not allow long-term predictions about intraguild predation. Ecological Applications 15 (4), 1111-1117.

Donovan, B.J., 1991. Life cycle of Sphecophaga vesparum (Curtis) (Hymenoptera: Ichneumonidae), a parasitoid of some vespid wasps. New Zeal and J. Zoology 18, 181-192.

Donovan, B.J., Read, P.E.C., 1987. Attempted biological control of social wasps, Vespula spp., (Hymenoptera: Ichneumonidae) in New Zealand. New Zealand J. Zoology 14, 329-335.

Dwyer, G., Dushoff, J., Elkinton, J.S., Levin, S.A., 2000. Pathogen-driven outbreaks in forest defoliators revisited: Building models from experimental data. Am. Nat. 156, 105-120.

Flint, M.L. (Ed.), 1987. Integrated Pest Management for Walnuts, University of California Statewide Integrated Pest Management Project, Division of Agriculture and Natural Resources, second edition. CA, publication 3270, University of California, Oakland, p. 3641.

Grasman, J., Van Herwaarden, O.A., Hemerik, L., Van Lenteren, J.C., 2001. A two-component model of host-parasitoid interactions: Determination of the size of inundative releases of parasitoids in biological pest control. Math Biosci. 169, 207-216.

Greathead, D.J., 1992. Natural enemies of tropical locusts and grasshoppers: Their impact and potential as biological control agents. In: Lomer, C.J., Prior, C. (Eds.), Biological Control of Locusts and Grasshoppers. C.A.B International, Wallingford, UK, pp. 105-121.

Hassell, M.P., 1978. The Dynamics of Arthopod Predator-Prey Systems. Princeton University Press, NJ.

Hassell, M.P., 1984. Insecticides in host-parasitoid interactions. Theor. Popul. Biol. 26, 378-386.

Hassell, M.P., 2000a. Host-parasitoid population dynamics. J. Anim. Ecol. 69 , 543-566.

Hassell, M.P., 2000b. The Spatial and Temporal Dynamics of Host-Parasitoid Interactions. Oxford University Press.

Hassell, M.P., May, R.M., 1973. Stability in insect host-parasite models. J. Anim. Ecol. 42, 693-726.

Hassell, M.P., May, R.M., 1974. Aggregation of predators and insect parasites and its effect on stability. J. Anim. Ecol. 43, 567-594. 
Hassell, M.P., Varley, G.C., 1969. New inductive population model for insect parasites and its bearing on biological control. Nature 223, 1133-1137.

Hastings, A., Higgins, K., 1994. Persistence of transitions in spatially structured ecological models. Science 263, 1133-1137.

Hawkins, B.A., Thomas, M.B., Hochberg, M.E., 1993. Refuge theory and biological control. Science 262, 1429-1432.

Headley, J.C., 1968. Estimating the productivity of agricultural pesticides. Amer. J. Agric. Econ. 50, 13-23.

Hoffmann, M.P., Frodsham, A.C., 1993. Natural enemies of vegetable insect pests. In: Cooperative Extension. Cornell University, Ithaca, NY, p. 63.

Lotka, A.J., 1920. Undamped oscillations derived from the law of mass action. J. Amer. Chem. Soc. 42, 1595-1599.

May, R.M., 1978. Host-parasitoid systems in patchy environments: A phenomenological model. J. Anim. Ecol. 47, 833-843.

May, R.M., 1985. Regulation of populations with non-overlapping generations by microparasites: A purely chaotic system. Am. Nat. 125, 573-585.

May, R.M., Hassell, M.P., 1988. Population dynamics and biological control. Philosophical Trans. Royal Society, London, Series B 318, 129-169.

May, R.M., Hassell, M.P., Anderson, R.M., Tonkyon, D.W., 1981. Density dependence in host-parasitoid models. J. Anim. Ecol. 50, 855-865.

McMurtry, J.A., Croft, B.A., 1997. Life styles of phytoseid mites and their roles in biological control. Ann. Rev. Entomol. 42, 291-321.

Metcalf, R.L., Luckman, W. (Eds.), 1975. Introduction to Insect Pest Management. John Wiley \& Sons, NY, p. 587.

Neuenschwander, P., Herren, H.R., 1988. Biological control of the Cassava Mealybug, Phenacoccus manihoti, by the exotic parasitoid Epidinocarsis lopezi in Africa. Philosophical Trans. Royal Soc. London. Series B, Biological Sciences 318, 319-333.

Nicholson, A.J., Bailey, V.A., 1935. The balance of animal populations. Part I. Proc. Zoological Soc. London 3, 551-598.

Parker, F.D., 1971. Management of pest populations by manipulating densities of both host and parasites through periodic releases. In: Huffaker, C.B. (Ed.), Biological Control. Plenum Press, New York.

Pedigo, L.P., 1996. Entomology and Pest Management, second edition. Prentice-Hall Pub., Englewood Cliffs, NJ, P679.

Pedigo, L.P., Higley, L.G., 1992. A new perspective of the economic injury level concept and environmental quality. Amer. Entomologist 38, 12-20.
Stone, L., Hart, D., 1999. Effects of immigration on the dynamics of simple population models. Theor. Popul. Biol. 55, 227-234.

Tang, S.Y., Chen, L.S., 2004. Modelling and analysis of integrated pest management strategy. Discrete Contin. Dyn. Syst. B 4, 759-768.

Tang, S.Y., Cheke, R.A., 2005. State-dependent impulsive models of integrated pest management (IPM) strategies and their dynamic consequences. J. Math. Biol. 50, 257-292.

Tang, S.Y., Xiao, Y.N., Chen, L.S., Cheke, R.A., 2005. Integrated pest management models and their dynamical behavior. Bull. Math. Biol. 67, $115-135$.

Udayagiri, S., Norton, A.P., Welter, S.C., 2000. Integrating pesticide effects with inundative biological control: Interpretation of pesticide toxicity curves for Anaphes iole in strawberries. Entomologia Experimentalis et Applicata 95, 87-95.

Van Dreische, R.E., Lyon, S., 2003. Commercial adoption of biological control-based IPM for whiteflies in pointsettia. Florida Entomologist 86, 481-483.

Van Lenteren, J.C., 1995. Integrated pest management in protected crops. In: Dent, D. (Ed.), Integrated Pest Management. Chapman \& Hall, London, pp. 311-320.

Van Lenteren, J.C., 2000. Measures of success in biological control of arthropods by augmentation of natural enemies. In: Wratten, S., Gurr, G. (Eds.), Measures of Success in Biological Control. Kluwer Academic Publishers, Dordrecht, pp. 77-89.

Van Lenteren, J.C., Woets, J., 1988. Biological and integrated pest control in greenhouses. Ann. Rev. Ent. 33, 239-250.

Varley, G.C., Gradwell, G.R., Hassell, M.P., 1973. Insect Population Ecology. Blackwell Scientific Publications, Oxford.

Volterra, V., 1931. Variations and fluctuations of a number of individuals in animal species living together. In: Chapman, R.N. (Ed.), Animal Ecology. McGraw Hill, New York, pp. 409-448.

Waage, J.K., Hassell, M.P., 1982. Parasitoids as biological control agents-a fundamental approach. Parasitology 84, 241-268.

Waage, J.K., Hassell, M.P., Godfray, H.C.J., 1985. The dynamics of pestparasitoid-insecticide interactions. J. Appl. Ecol. 22, 825-838.

White, K.A.J., Wilson, K., 1999. Modelling density-dependent resistance in insect-pathogen interactions. Theor. Popul. Biol. 56, 163-181. 